\title{
Loading as a design parameter for genetic circuits
}

by

Nithin Senthur Kumar

B.E., Vanderbilt University (2014)

Submitted to the Department of Mechanical Engineering in partial fulfillment of the requirements for the degree of

$$
\begin{aligned}
& \text { Master of Science } \\
& \text { at the } \\
& \text { MASSACHUSETTS INSTITUTE OF TECHNOLOGY }
\end{aligned}
$$

June 2016

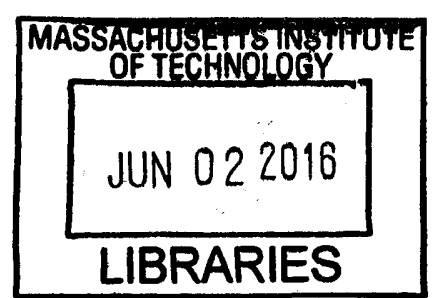

ARCHIVES

(C) Massachusetts Institute of Technology 2016. All rights reserved.

Author .........

\section{Signature redacted}

Department of Mechanical Engineering

Certified by .................

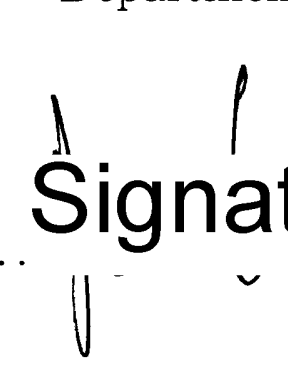

Accepted by

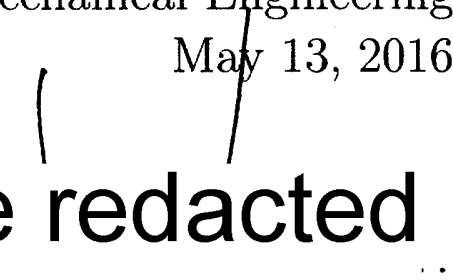

Domitilla Del Vecchio Associate Professor Thesis Supervisor

Signature redacted
Rŏhan Abeyaratne Chairman, Department Committee on Graduate Theses 


\section{DISCLAIMER NOTICE}

Due to the condition of the original material, there are unavoidable flaws in this reproduction. We have made every effort possible to provide you with the best copy available.

Thank you.

The images contained in this document are of the best quality available. 


\title{
Loading as a design parameter for genetic circuits
}

\author{
by \\ Nithin Senthur Kumar \\ Submitted to the Department of Mechanical Engineering \\ on May 13, 2016, in partial fulfillment of the \\ requirements for the degree of \\ Master of Science
}

\begin{abstract}
Since the 2000s, there have been several forms of synthetic genetic circuits that have been modeled and experimentally validated. Examples include the toggle switch, repressilator, various configurations of oscillators, and even logic gates. A major goal in synthetic biology is to combine these modules to construct complex circuits for applications including biosensing, biofuel technology, and various medical technologies. However, a significant problem when building complex biomolecular circuits is due to context-dependence: the dynamics of a system are altered upon changes to its context, potentially degrading the system's performance.

In this thesis, we study retroactivity, a specific type of context-dependence, by analyzing the effects of loads on a transcription factor applied by the transcription factor's target sites. In particular, we study this loading effect on the model of an activator-repressor oscillator, a widely studied motif in systems and systems biology. Our analysis indicates that strong activation and weak repression are key for a stable limit cycle. Repression can be effectively weakened by adding load to the repressor, while activation can be effectively weakened by adding load to the activator. Therefore, loading the repressor can be employed as a design parameter to establish a stable limit cycle. In contrast, loading the activator is deleterious to the clock. Experimental protocol and preliminary data are presented. The results presented in this thesis may be helpful to provide a method to tune the dynamics of synthetic genetic circuits without using tags and modifying promoter regions.
\end{abstract}

Thesis Supervisor: Domitilla Del Vecchio

Title: Associate Professor 


\section{Acknowledgments}

I would like to thank my advisor, Prof. Domitilla Del Vecchio for not only introducing me to this vibrant research field, but also for her patient guidance while I initially came to terms with working on biomolecular systems. Her enthusiasm for her work is inspirational and I am truly fortunate to be mentored by such a remarkable individual.

It would be remiss to not thank Prof. Eric Barth, Mark Hofacker, and Alexander Pedchenko for initially introducing me to research and subtly convincing me that graduate school would ultimately be a rewarding experience.

My labmates have all been extremely helpful, especially Dr. Hsin-Ho Huang, for introducing and instructing me on how to use the wet lab, and Yili Qian, for his insightful feedback on my papers and presentations. I would also like to thank Dr. Abdullah Hamadeh for patiently reading and editing my initial research notes.

My parents, Velumathi Kumar and Sivanappan Kumar, have had to cope with their only child studying on the opposite end of the planet for six years and yet their unconditional support has never wavered. If I could dedicate this thesis to anyone, it would be to you two.

This work was supported by NIH P50 GM098792. 


\section{Contents}

$\begin{array}{lll}1 & \text { Introduction } & 15\end{array}$

1.1 Modularity . . . . . . . . . . . . . . . . . 15

1.2 Motivating example . . . . . . . . . . . . . 16

1.3 Thesis content . . . . . . . . . . . . . . . . 18

2 Model derivation $\quad 21$

2.1 Model . . . . . . . . . . . . . . . . . . . . . . . . 21

2.2 Two-state approximation $\ldots \ldots \ldots \ldots \ldots \ldots \ldots \ldots$

3 Oscillator with downstream load $\quad 29$

3.1 Downstream load to A . . . . . . . . . . . . . . . . . . 29

3.2 Downstream load to $\mathrm{R} \ldots \ldots \ldots \ldots \ldots \ldots \ldots$

4 Oscillator with downstream load and decay $\quad 35$

4.1 Complex decay to $\mathrm{A} \ldots \ldots \ldots \ldots \ldots$

4.2 Complex decay to $\mathrm{R} \ldots \ldots \ldots \ldots \ldots \ldots \ldots$

5 Experimental Protocol Development 39

5.1 Introduction . . . . . . . . . . . . . . . . . . 39

5.2 Plasmid and strain $\ldots \ldots \ldots \ldots \ldots \ldots \ldots$

5.3 Trapping cells $\ldots \ldots \ldots \ldots \ldots \ldots \ldots \ldots$

5.3 .1 ConA protocol $\ldots \ldots \ldots \ldots \ldots \ldots \ldots$

$5.3 .2 \quad$ Agarose pad protocol . . . . . . . . . . . . . . 43

5.3 .3 Microfluidics protocol . . . . . . . . . . . . . . 43 
5.4 Fluorescence data . . . . . . . . . . . . . . 46

6 Conclusion and Future Work $\quad 49$

A Four-State Model Derivation of Activator-Repressor Genetic Oscillator $\quad 51$

B Model Reduction via Singular Perturbation for Two-State System $\begin{array}{ll}\text { with downstream load } & 55\end{array}$

C Model Reduction via Singular Perturbation for Two-State System with downstream load and complex decay $\quad 57$

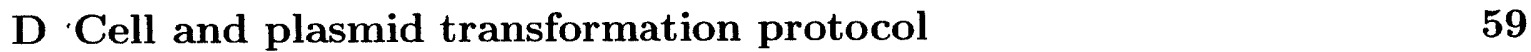

$\begin{array}{lll}\text { E PCB design and construction protocol } & 61\end{array}$

F Fluorescence calculation $\quad 63$ 


\section{List of Figures}

1-1 Diagram of system considered in motivating example. The upstream system that produces protein $A$ is regulated by $X . X$ binds to the promoter site, $P_{a}$, which initiates transcription of the gene $a$, leading to the production of protein $A$. Protein $A$ then acts as a transcription factor to 5 downstream modules. For a sinusoidal input $X$, the ideal system responses from all modules are also sinusoids as shown in the inset next to each protein. . . . . . . . . . . .

1-2 Simulation of two systems described in equation (1.7). The system response for the isolated system (black), 1 downstream system (blue), and 5 downstream systems (red) for input, $X(t)=1+\sin (0.5 t)$. Simulation parameters include: $\kappa_{I}=10 h r^{-1}, \delta_{I}=1 h r^{-1}, f_{I}=1, r_{I}=1, I=$ $B, C, D, E, F . \quad \ldots \ldots \ldots \ldots \ldots \ldots \ldots \ldots \ldots \ldots$

2-1 Diagram of a synthetic genetic oscillator. Genes a and $\mathrm{r}$ are translated by RNA polymerase (not shown) to produce the mRNA strands, $\mathrm{m}_{\mathrm{A}}$ and $m_{R}$. Ribosomes then produce proteins $A$ and $R$ by linking amino acids in the order specified by $\mathrm{m}_{\mathrm{A}}$ and $\mathrm{m}_{\mathrm{R}}$, respectively. A and $\mathrm{R}$ multimerize to form $A_{2}$ and $R_{4}$, respectively. $A_{2}$ transcriptionally activates a and $\mathrm{r}$ by binding to their DNA promoter sites, while $\mathrm{R}_{4}$ transcriptionally represses a and $\mathrm{r}$ by competitively binding to the same sites. Only the complex formed by $\mathrm{A}_{2}$ and the DNA promoter can initiate transcription. Activation is denoted by an arrow and repression is denoted by a bar arrow. . . . . . . . . . . . . . . . 
2-2 Diagram of a two-state activator-repressor genetic oscillator. Proteins $\mathrm{A}$ and $\mathrm{R}$ directly activate and repress themselves and each other, respectively, and mRNA dynamics are assumed to be at quasi-steady state. . . . . . . . . . . . . . . . . .

2-3 System nullclines for (2.4)-(2.5) demonstrating the change in number of equilibria from 3 (a with closeup c) to 1 (b with closeup d) as $P(0)$ is made more negative (i.e., for sufficiently large $\alpha_{0}$, the system has 1 equilibrium point). System parameter values include: $\alpha=20 \mathrm{hr}^{-1} /$ gene, $k_{A}=k_{R}=1 \mathrm{nM}, \alpha_{0}=0.05(\mathbf{a c}), 2(\mathbf{b d}) \mathrm{nMhr}^{-1} /$ gene, $\delta_{A}=\delta_{R}=1 \mathrm{hr}^{-1}$, $\kappa_{A}=2 \mathrm{hr}^{-1}, \kappa_{R}=1 \mathrm{hr}^{-1}, \gamma_{A}=4 \mathrm{hr}^{-1}, \gamma_{R}=0.5 \mathrm{hr}^{-1}, n=2, m=4 . .$.

2-4 Four possible nullcline intersection regions of (2.4) and (2.5) given by blue solid $(f=0)$ and green dotted lines $(g=0)$, respectively. System parameters include $\alpha=20 \mathrm{nMhr}^{-1}, k_{A}=k_{R}=1 \mathrm{nM}, \alpha_{0}=40(\mathbf{a}), 2(\mathbf{b})$, $0.1(\mathbf{c}), 2(\mathbf{d}) \mathrm{nMhr}^{-1}, \delta_{A}=\delta_{R}=1 \mathrm{hr}^{-1}, \kappa_{A}=\kappa_{R}=1 \mathrm{hr}^{-1}, \gamma_{A}=2 \mathrm{hr}^{-1}$, $\gamma_{R}=1(\mathbf{a b d}), 10(\mathbf{c}) \mathrm{hr}^{-1}, n=2(\mathbf{a b c}) 1(\mathbf{d}), m=2(\mathbf{a b c}) 1(\mathbf{d}) . \ldots$

2-5 Change in response of $\mathrm{A}$ from decaying to stable oscillations as $\kappa_{A}$ increases for system given by (2.4)-(2.5). System parameters include $\alpha=1200 \mathrm{hr}^{-1} /$ gene, $k_{A}=k_{R}=1 \mathrm{nM}, \alpha_{0}=120 \mathrm{hr}^{-1} /$ gene, $\delta_{A}=\delta_{R}=$ $10 \mathrm{hr}^{-1}, \kappa_{R}=50 \mathrm{hr}^{-1}, \gamma_{A}=3.3 \mathrm{hr}^{-1}, \gamma_{R}=1 \mathrm{hr}^{-1}, \mathrm{n}=2, \mathrm{~m}=2 . \ldots$.

3-1 Load to A by downstream promoter sites $C_{10}$. The original two-state oscillator system is given in the dashed box. . . . . . . . . .

3-2 Simulation of (3.1) with system parameters: $\alpha=1200 \mathrm{hr}^{-1} /$ gene, $k_{A}=$ $k_{R}=1 \mathrm{nM}, \alpha_{0}=120 \mathrm{hr}^{-1} /$ gene, $\delta_{A}=\delta_{R}=10 \mathrm{hr}^{-1}, \kappa_{A}=\kappa_{R}=50 \mathrm{hr}^{-1}$, $\gamma_{A}=3.5 \mathrm{hr}^{-1}, \gamma_{R}=1 \mathrm{hr}^{-1}, \mathrm{n}=2, \mathrm{~m}=2 \ldots \ldots \ldots$

3-3 Load to $\mathrm{R}$ by downstream promoter sites $C_{20}$. The original two-state oscillator system is given in the dashed box. . . . . . . . . . . 
3-4 Simulation of (3.3) demonstrating the transition from decayed response to oscillatory behavior by addition of downstream $C_{t 2}=1000 \mathrm{nM}$ at $t=100 \mathrm{hr}$. System parameters include: $\alpha=1200 \mathrm{hr}^{-1} /$ gene, $k_{A}=$ $k_{R}=1 \mathrm{nM}, \alpha_{0}=120 \mathrm{hr}^{-1} /$ gene, $\delta_{A}=\delta_{R}=10 \mathrm{hr}^{-1}, \kappa_{A}=\kappa_{R}=50 \mathrm{hr}^{-1}$, $\gamma_{A}=3.3 \mathrm{hr}^{-1}, \gamma_{R}=1 \mathrm{hr}^{-1}, \mathrm{n}=2, \mathrm{~m}=2 \ldots \ldots \ldots$

4-1 Bifurcation diagram of system described by (4.2). As the bifurcation parameter, $C_{t 1}$ is increased, both systems undergo a subcritical Hopf bifurcation. System parameters include $\alpha=1200 \mathrm{hr}^{-1} /$ gene, $k_{A}=$ $k_{R}=1 \mathrm{nM}, \alpha_{0}=120 \mathrm{hr}^{-1} /$ gene, $\delta_{A}=\delta_{R}=10 \mathrm{hr}^{-1}, \kappa_{A}=\kappa_{R}=50 \mathrm{hr}^{-1}$, $\gamma_{A}=3.4 \mathrm{hr}^{-1}, \gamma_{R}=1 \mathrm{hr}^{-1}, \mathrm{n}=2, \mathrm{~m}=2, K_{d 1}=1, \pi_{A}=1 \mathrm{hr}^{-1} \ldots . .$.

4-2 Bifurcation diagram of system described by (4.3). As the bifurcation parameter, $C_{t 2}$, is increased, the system with only load undergoes a supercritical Hopf bifurcation while the system with decay is always stable. System parameters include $\alpha=1200 \mathrm{hr}^{-1} /$ gene, $k_{A}=k_{R}=1 \mathrm{nM}$, $\alpha_{0}=120 \mathrm{hr}^{-1} /$ gene, $\delta_{A}=\delta_{R}=10 \mathrm{hr}^{-1}, \kappa_{A}=\kappa_{R}=50 \mathrm{hr}^{-1}, \gamma_{A}=3.3 \mathrm{hr}^{-1}$, $\gamma_{R}=1 \mathrm{hr}^{-1}, \mathrm{n}=2, \mathrm{~m}=2, K_{d 2}=1, \pi_{R}=1 \mathrm{hr}^{-1} \ldots \ldots \ldots$

5-1 Plasmids pJS167 and pJS169 that contain the activator-repressor oscillator. Image obtained from $[9] . \ldots \ldots \ldots$

5-2 Schematic of oscillator circuit construction. The plasmids, pJS167 (red circle) and pJS169 (green triangle), are originally in the DH5- $\alpha$ strain (blue oval) and are purified using the Zymo Research ZR Plasmid Miniprep Kit. The host cell (dashed orange oval) is made competent and transformed (see Appendix D for protocol) with the extracted plasmids to yield the oscillator circuit. . . . . . . . . . .

5-3 Time-lapse bright-field microscopy using ConA protocol. Interval between images is $12 \mathrm{~min}$. After replication (third frame), the daughter cell is not constrained by the chamber wall and disappears from the frame of view. . . . . . . . . . . . . . . . . 
5-4 Side view of experiment setup with the agarose pad protocol. The cover glass (black lines) are placed on either side of the perfusion chamber (orange), which is filled with the agar gel (light pink). The cells (green) are sandwiched between the top layer of the gel and the top cover glass. This diagram intentionally exaggerates the space between the agar gel and the top cover glass to demonstrate that there is sufficient space for the cells to traverse despite firmly attaching the cover glass to the chamber. . . . . . . . . . . . . . . . .

5-5 Left: PCB design (credit: Dr. Hsin-Ho Huang) overlaid with CellASIC ONIX microfluidic plate schematic. Right: Black wires correspond to the copper wires in the actual board (fabricated by Dr. Hsin-Ho Huang and Nithin Kumar) . . . . . . . . . . . . . . . .

5-6 Left: Experimental setup including pump (purple, left), heater controller (white, right) with $\mathrm{PCB}$ heater affixed onto the microfluidic plate (center), which rests on the microscope stage. Right: Close-up of PCB heater and microfluidic plate with sealed cell culture suspension (clear, plastic tube on the right) and thermocouple (white wire) for heater controller. . . . . . . . . . . . . . .

5-7 Single-cell fluorescence trajectories for 5 randomly selected cells for 5

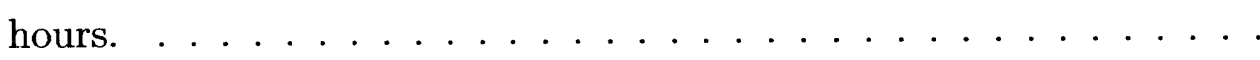

5-8 Time-lapse bright-field and green fluorescence images of oscillator using the microfluidic protocol. Images are at $30 \mathrm{~min}$ intervals (i.e., top left pair of images are bright-field, left, and fluorescence, right, at $t=0 \mathrm{~min}$ ) for a total time of $5 \mathrm{hrs} . \ldots \ldots \ldots \ldots$ 


\section{List of Tables}

F.1 Cell 1 data. . . . . . . . . . . . . . . . 65

F.2 Cell 2 data. . . . . . . . . . . . . . . . 68

F.3 Cell 3 data. . . . . . . . . . . . . . . . . 70

F.4 Cell 4 data. . . . . . . . . . . . . . . . . 72

F.5 Cell 5 data. . . . . . . . . . . . . . . 74 


\section{Chapter 1}

\section{Introduction}

\subsection{Modularity}

Modularity is the property that allows components to be designed independently such that their input/output behavior remains unchanged upon interconnection with other modules. Modularity is, however, not universal and the dynamics of engineering systems typically change when loaded. Recently, it has been experimentally shown that biomolecular systems, such as genetic circuits, experience these loading effects [1]-[2], also called retroactivity [3]. Several types of simple biomolecular circuits and motifs, such as the toggle switch [4]-[6], gene oscillators [7]-[9], and logic gates [10][11] have been modularly designed and experimentally validated. A major challenge in synthetic biology is to combine these modules to construct complex circuits [12] for applications including biofuel technology [13], biosensors [14], developing vaccines [15]-[16], and other medical technologies [17]-[18].

To address the deleterious effects of retroactivity that cause modularity to fail, insulation devices have been designed to be placed between upstream and downstream systems to act as a buffer. Such devices include using high gain negative feedback [3], [19] and time scale separation [2], [20]. However, retroactivity can be viewed as

an additional design parameter and in fact, all natural systems have some form of load. Signal transduction networks often regulate many downstream gene targets and in gene transcription networks, transcription factors often have many DNA sites to 
which they bind. Many of these sites do not even have regulatory functionalities [21], and therefore it is plausible that they are being used to tune the level and temporal dynamics of transcription factors [1].

Recent theoretical work has considered the effect of loading on synthetic biological circuits and networks. It has been theoretically shown that loading a genetic clock through the use of additional DNA promoter binding sites can switch it on or off and also enable frequency tuning [21]. Retroactivity has been shown to affect the relative stability of toggle switches, enabling the engineering of biased switches [22]. The loading effects due to intramodular and intermodular connections leading to internal, scaling, and mixing retroactivity are studied in [23]. In this thesis, we consider the activator-repressor clock built in [9]. In particular, we identify parametric conditions

for the existence of a stable limit cycle and analyze the effect of load on the clock's dynamics with regard to activating and quenching oscillations using standard tools from dynamical systems theory.

\subsection{Motivating example}

To demonstrate how retroactivity complicates the modular design of genetic circuits, suppose one wished to regulate five transcriptional modules by a single module (as shown in Fig. 1-1). The upstream controller module is regulated by a transcription factor, $X$, which binds to the promoter site, $P_{a}$ of gene $a$, which (after transcription and translation) produces the protein, $A$. The five downstream systems are then regulated by $A$, which now acts as a transcription factor by binding to their promoter site, $P_{i}$, of gene $i$, (where $i=b, c, d, e, f$ ) and produces their respective protein. If one were to independently design these modules without taking into account the interconnection between the systems, the reactions for the upstream module would 


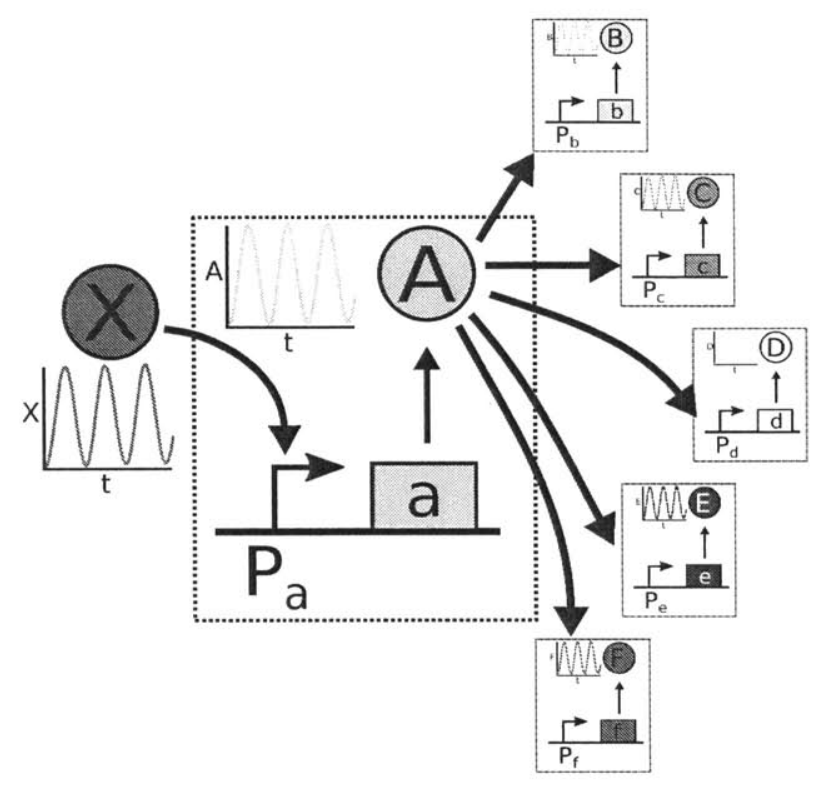

Figure 1-1: Diagram of system considered in motivating example. The upstream system that produces protein $A$ is regulated by $X$. $X$ binds to the promoter site, $P_{a}$, which initiates transcription of the gene $a$, leading to the production of protein $A$. Protein $A$ then acts as a transcription factor to 5 downstream modules. For a sinusoidal input $X$, the ideal system responses from all modules are also sinusoids as shown in the inset next to each protein.

be given as:

$$
\begin{aligned}
\mathrm{X}+\mathrm{P}_{\mathrm{a}} \stackrel{\mathrm{f}_{\mathrm{a}}}{\underset{\mathrm{r}_{\mathrm{a}}}{\rightleftharpoons}} \mathrm{C}_{\mathrm{a}}, \\
\mathrm{C}_{\mathrm{a}} \stackrel{\kappa_{a}}{\longrightarrow} \mathrm{C}_{\mathrm{a}}+\mathrm{A}, \\
\mathrm{A} \stackrel{\delta_{a}}{\longrightarrow} \emptyset .
\end{aligned}
$$

Reaction (1.1) describes the reversible binding reaction of $X$ and the promoter site to form a complex $C_{a}$, with forward rate $f_{a}$ and reverse rate $r_{a}$. Reaction (1.2) lumps the dynamics of transcription and translation into a constant rate, $\kappa_{a}$, leading to the production of $A$ and reaction (1.3) describes the decay of $A$ at a rate $\delta_{a}$. Similarly, 
the reactions for the downstream systems are given by:

$$
\begin{aligned}
\mathrm{A}+\mathrm{P}_{\mathrm{i}} \stackrel{\mathrm{f}_{\mathrm{i}}}{\underset{\mathrm{r}_{\mathrm{i}}}{\rightleftharpoons}} \mathrm{C}_{\mathrm{i}}, \\
\mathrm{C}_{\mathrm{i}} \stackrel{\kappa_{i}}{\longrightarrow} \mathrm{C}_{\mathrm{i}}+\mathrm{I}, \\
\mathrm{I} \stackrel{\delta_{i}}{\longrightarrow} \emptyset,
\end{aligned}
$$

where $I$ denotes protein from a particular module $(I=B, C, D, E, F)$. The dynamics of $A$ in the isolated system, $A_{\text {iso }}$, and the connected system, $A_{\text {con }}$, are given by:

$$
\begin{aligned}
& \dot{A}_{i s o}=\kappa_{A} C_{A}-\delta_{A} A \\
& \dot{A}_{c o n}=\kappa_{A} C_{A}-\delta_{A} A+\sum_{i=b}^{f}\left(r_{i} C_{i}-f_{i} A P_{i}\right)
\end{aligned}
$$

The $\sum_{i=b}^{f}\left(r_{i} C_{i}-f_{i} A P_{i}\right)$ term is the effect of the retroactivity applied by the downstream modules to the output of the $A$ module and is absent in the isolated system. To see how it modifies the dynamics of $A$, consider a sinusoidal input, $X$ (as shown in Fig. 1-1). The response of $A$ when isolated, connected to just 1 downstream module (say $B$ ) and 5 downstream modules is given in Fig. 1-2. We find that load to $A$ decreases its oscillation amplitude, a characteristic not desirable for a regulatory module (i.e., a parent module should ideally not change its dynamics when connected to daughter modules). In this work, however, we show that loading is not necessarily detrimental to the performance of genetic circuits and in fact can be used to tune the dynamics of an activator-repressor oscillator circuit.

\subsection{Thesis content}

This paper is organized as follows. In Chapter 2, we derive a deterministic ODE model of the activator-repressor clock from biochemical reactions and provide conditions for the existence of a stable limit cycle. In Chapter 3, the effect of load to the activation and repression branches on the dynamics of the oscillator, respectively is studied. 


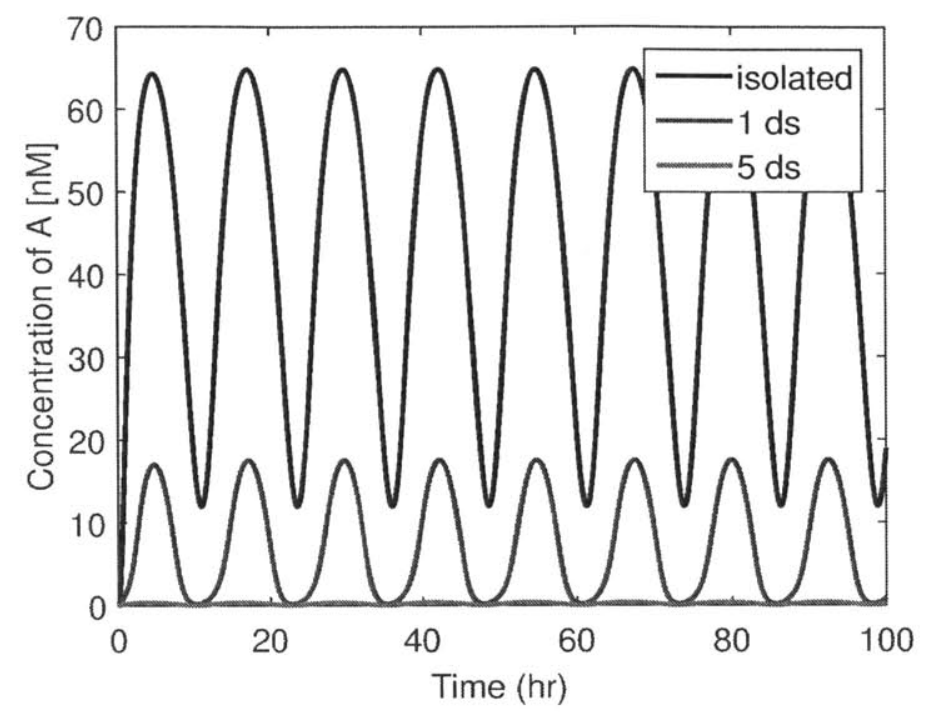

Figure 1-2: Simulation of two systems described in equation (1.7). The system response for the isolated system (black), 1 downstream system (blue), and 5 downstream systems (red) for input, $X(t)=1+\sin (0.5 t)$. Simulation parameters include: $\kappa_{I}=10 h r^{-1}, \delta_{I}=1 h r^{-1}, f_{I}=1, r_{I}=1, I=B, C, D, E, F$.

In Chapter 4, complex decay of transcription factor bound with DNA promoter is considered and the qualitative change in system behavior is studied. In Chapter 5, three experimental protocols are presented and preliminary experimental results are discussed. 


\section{Chapter 2}

\section{Model derivation}

\section{$2.1 \quad$ Model}

A representation of the core dynamics of the genetic oscillator described in [9] is given in Fig. 2-1. The genes a and $r$ undergo transcription to produce the mRNA of the activator protein $(\mathrm{A})$, denoted by $\mathrm{m}_{\mathrm{A}}$, and the repressor protein $(\mathrm{R})$, denoted by $\mathrm{m}_{\mathrm{R}}$, respectively. Ribosomes (shown in green) decode $m_{A}$ and $m_{R}$ into amino acid chains that fold to form the protein molecules, $A$ and $R$, respectively. The proteins then multimerize to form $\mathrm{A}_{2}$ and $\mathrm{R}_{4}$. $\mathrm{A}_{2}$ positively regulates its own production by binding to its own promoter site (left of a) to initiate transcription and the production of $\mathrm{R}$ by similarly binding to its promoter site (left of $\mathrm{r}$ ). Conversely, $\mathrm{R}_{4}$ negatively regulates its own production by competitively binding to the same promoter sites. Unlike the complex of $\mathrm{A}_{2}$ and the promoter site, the complex of $\mathrm{R}_{4}$ and the promoter site does not initiate transcription and so, $R_{4}$ essentially sequesters free DNA promoter sites.

A deterministic ODE model can be derived from considering the biochemical reactions of activation, repression, transcription, translation, and multimerization and be used to describe the dynamics of the concentration of both $A$ and $R$ and is given 


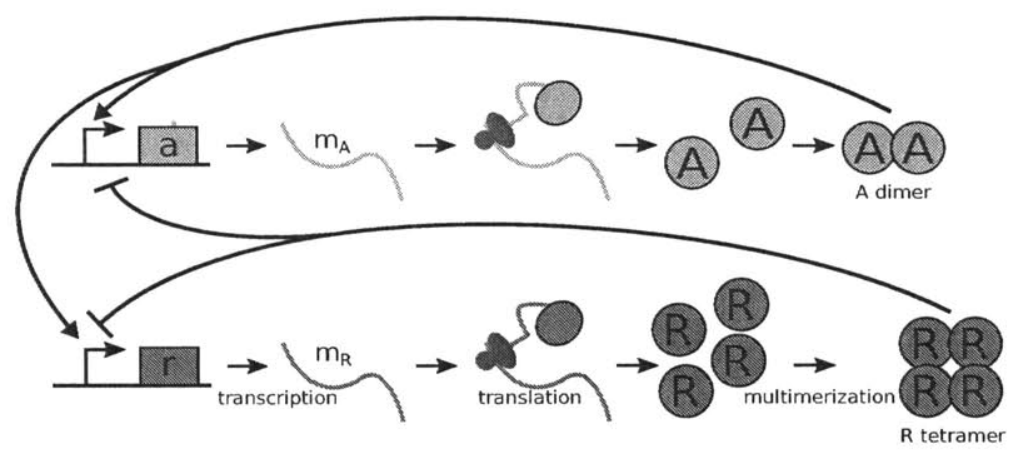

Figure 2-1: Diagram of a synthetic genetic oscillator. Genes a and $\mathrm{r}$ are translated by RNA polymerase (not shown) to produce the mRNA strands, $m_{A}$ and $m_{R}$. Ribosomes then produce proteins $\mathrm{A}$ and $\mathrm{R}$ by linking amino acids in the order specified by $\mathrm{m}_{\mathrm{A}}$ and $m_{R}$, respectively. $A$ and $R$ multimerize to form $A_{2}$ and $R_{4}$, respectively. $A_{2}$ transcriptionally activates a and $\mathrm{r}$ by binding to their DNA promoter sites, while $\mathrm{R}_{4}$ transcriptionally represses a and $\mathrm{r}$ by competitively binding to the same sites. Only the complex formed by $\mathrm{A}_{2}$ and the DNA promoter can initiate transcription. Activation is denoted by an arrow and repression is denoted by a bar arrow.

by (see Appendix A for details):

$$
\begin{aligned}
\dot{m}_{A} & =\frac{\alpha\left(A / k_{A}\right)^{n}+\alpha_{0}}{1+\left(A / k_{A}\right)^{n}+\left(R / k_{R}\right)^{m}}-\delta_{A} m_{A}, \\
\dot{m}_{R} & =\frac{\alpha\left(A / k_{A}\right)^{n}+\alpha_{0}}{1+\left(A / k_{A}\right)^{n}+\left(R / k_{R}\right)^{m}}-\delta_{R} m_{R}, \\
\dot{A} & =\kappa_{A} m_{A}-\gamma_{A} A \\
\dot{R} & =\kappa_{R} m_{R}-\gamma_{R} R .
\end{aligned}
$$

The parameter $\alpha$ is a measure of the maximum transcriptional activation by $A_{n}: D^{P} A^{P}$ to mRNA and $\alpha_{0}$ represents transcriptional leakiness (or basal transcriptional expression) of mRNA. Since the promoters controlling the expression of A and R are the same, given the symmetry of the system, we can assume that $\alpha$ and $\alpha_{0}$ are equal for both the $\mathrm{m}_{\mathrm{A}}$ and $\mathrm{m}_{\mathrm{R}}$ dynamics. The contribution of basal transcription to the production of mRNA is assumed to be small in magnitude compared to that of the promoter (i.e., $\frac{\alpha_{0}}{\alpha}<<1$ ). The parameters $k_{A}$ and $k_{R}$ indicate the relative affinity for complex formation and multimerization. The protein translation and decay rate are given by $\kappa$ and $\gamma$, respectively and the mRNA decay rate is denoted by $\delta$. 


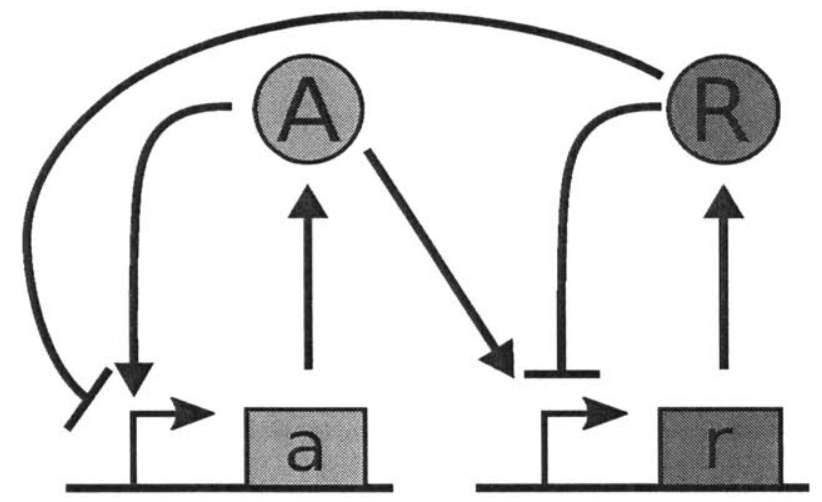

Figure 2-2: Diagram of a two-state activator-repressor genetic oscillator. Proteins $\mathrm{A}$ and $\mathrm{R}$ directly activate and repress themselves and each other, respectively, and mRNA dynamics are assumed to be at quasi-steady state.

\subsection{Two-state approximation}

Due to the time scale separation between transcription of DNA to mRNA and translation, the original four-state system (2.1) can be reduced to a two-state system by considering $m_{A}$ and $m_{R}$ at their quasi-steady state [25]:

$$
\begin{aligned}
& m_{A}=\frac{1}{\delta_{A}} \frac{\alpha\left(A / k_{A}\right)^{n}+\alpha_{0}}{1+\left(A / k_{A}\right)^{n}+\left(R / k_{R}\right)^{m}}, \\
& m_{R}=\frac{1}{\delta_{R}} \frac{\alpha\left(A / k_{A}\right)^{n}+\alpha_{0}}{1+\left(A / k_{A}\right)^{n}+\left(R / k_{R}\right)^{m}} .
\end{aligned}
$$

Substituting (2.2)-(2.3) in the $\dot{A}, \dot{R}$ equations of (2.1) yields the two-state approximation (pictorially represented in Fig. 2-2):

$$
\begin{aligned}
& \dot{A}=\frac{\kappa_{A}}{\delta_{A}} \frac{\alpha\left(A / k_{A}\right)^{n}+\alpha_{0}}{1+\left(A / k_{A}\right)^{n}+\left(R / k_{R}\right)^{m}}-\gamma_{A} A=f(A, R), \\
& \dot{R}=\frac{\kappa_{R}}{\delta_{R}} \frac{\alpha\left(A / k_{A}\right)^{n}+\alpha_{0}}{1+\left(A / k_{A}\right)^{n}+\left(R / k_{R}\right)^{m}}-\gamma_{R} R=g(A, R) .
\end{aligned}
$$

To determine sufficient conditions for oscillatory behavior, the Poincaré-Bendixson theorem [26] is used to infer the existence of a stable limit cycle. This requires (i) the existence of a unique equilibrium point and (ii) that this equilibrium point is unstable and not a saddle point.

Since the system is two-dimensional, analyzing the expression for the nullclines 
provides a convenient way of determining conditions for a unique equilibrium point, given by $\left(A_{e}, R_{e}\right)$. The nullclines are defined by setting $\dot{A}=0$ and $\dot{R}=0$ and are given by:

$$
\begin{aligned}
& R_{e}=k_{R}\left[\frac{\kappa_{A}}{\delta_{A} \gamma_{A} A_{e}}\left[\alpha\left(\frac{A_{e}}{k_{A}}\right)^{n}+\alpha_{0}\right]-\left(1+\left(\frac{A_{e}}{k_{A}}\right)^{n}\right)\right]^{\frac{1}{m}} \\
& A_{e}=k_{A}\left[\frac{-\kappa_{R} \alpha_{0}+\gamma_{R} \delta_{R} R_{e}\left(1+\left(\frac{R_{e}}{k_{R}}\right)^{m}\right)}{\kappa_{R} \alpha-\gamma_{R} \delta_{R} R_{e}}\right]^{\frac{1}{n}}
\end{aligned}
$$

Claim: If $\alpha_{0}$ is sufficiently large, system (2.4)-(2.5) has a unique equilibrium point.

Proof. Setting $\dot{A}=0, \dot{R}=0$ in (2.4)-(2.5), solving for $\frac{\alpha\left(A / k_{A}\right)^{n}+\alpha_{0}}{1+\left(A / k_{A}\right)^{n}+\left(R / k_{R}\right)^{m}}$, and equating the resulting expressions yields:

$$
\frac{\kappa_{A}}{\delta_{A} \gamma_{A} A_{e}}=\frac{\kappa_{R}}{\delta_{R} \gamma_{R} R_{e}}
$$

This results in the equilibrium point lying on the line defined by:

$$
A_{e}=c R_{e}, \quad c=\frac{\kappa_{A} \delta_{R} \gamma_{R}}{\kappa_{R} \delta_{A} \gamma_{A}}
$$

Substituting (2.9) in (2.7) yields:

$P\left(R_{e}\right)=\left(\frac{k_{A}^{n} \gamma_{R} \delta_{R}}{k_{R}^{m}}\right) R_{e}^{m+1}+\left(\gamma_{R} \delta_{R} c^{n}\right) R_{e}^{n+1}-\left(\kappa_{R} \alpha c^{n}\right) R_{e}^{n}+\left(k_{A}^{n} \gamma_{R} \delta_{R}\right) R_{e}-k_{A}^{n} \kappa_{R} \alpha_{0}=0$

For $n, m>1$, the coefficients of $P\left(R_{e}\right)$ change signs three times when arranged in descending powers of $R_{e}$ (regardless of the value of $n$ or $m$ ). By Descartes' rule of signs [27], $P\left(R_{e}\right)=0$ has either one or three positive root(s). Since the coefficient of the terms with the largest exponent (either $m+1$ or $n+1$ ) is always positive, as $R_{e} \rightarrow \infty, P\left(R_{e}\right) \rightarrow \infty$. To ensure a single positive root, it is enough to translate $P\left(R_{e}\right)$ sufficiently vertically downwards.

To this end, let $R_{\mathrm{M}}$ be any value of $R_{e}$ sufficiently large such that there are no 

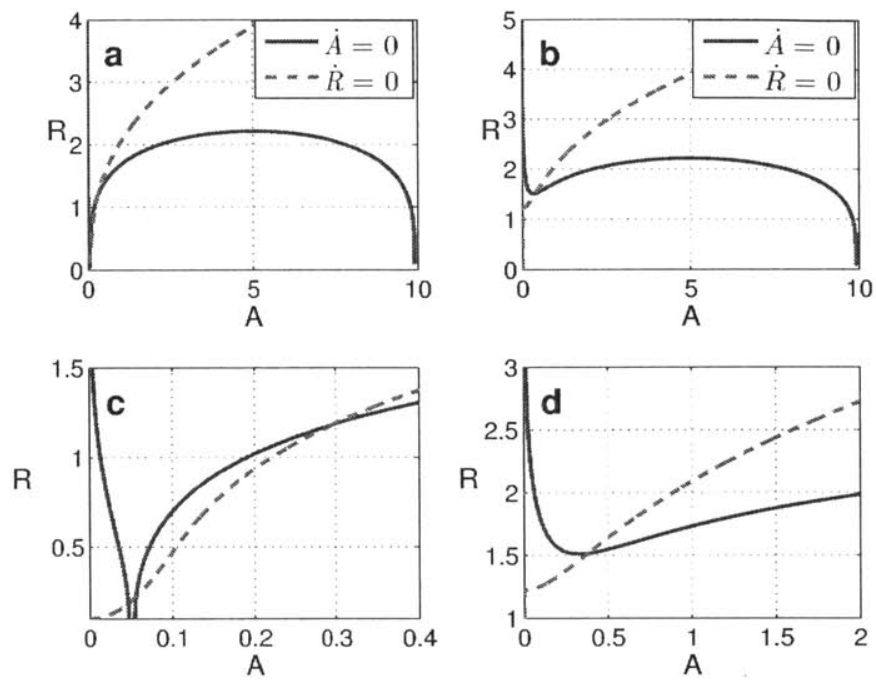

Figure 2-3: System nullclines for (2.4)-(2.5) demonstrating the change in number of equilibria from 3 (a with closeup c) to 1 (b with closeup $\mathbf{d}$ ) as $P(0)$ is made more negative (i.e., for sufficiently large $\alpha_{0}$, the system has 1 equilibrium point). System parameter values include: $\alpha=20 \mathrm{hr}^{-1} /$ gene, $k_{A}=k_{R}=1 \mathrm{nM}, \alpha_{0}=0.05(\mathbf{a c}), 2(\mathbf{b d})$ $\mathrm{nMhr}^{-1} /$ gene, $\delta_{A}=\delta_{R}=1 \mathrm{hr}^{-1}, \kappa_{A}=2 \mathrm{hr}^{-1}, \kappa_{R}=1 \mathrm{hr}^{-1}, \gamma_{A}=4 \mathrm{hr}^{-1}, \gamma_{R}=0.5 \mathrm{hr}^{-1}$, $n=2, m=4$.

more inflection points for $P\left(R_{e}>R_{M}\right)$ and let $M=\sup _{R_{e} \in\left[0, R_{M}\right]} P\left(R_{e}\right)$. Since $P\left(R_{e}\right)$ is a real-valued, continuous function of $R_{e}$ in the closed interval $\left[0, R_{M}\right]$, by the Extreme Value Theorem [28], $M$ must be finite.

The parameter, $\alpha_{0}$, which corresponds to the basal expression rate of mRNA does not appear in any of the coefficients of $P\left(R_{e}\right)$ except for the constant term. Therefore, increasing $\alpha_{0}$ translates $P\left(R_{e}\right)$ downwards and so for any $M, P(0)$ can be set sufficiently negative so that $P\left(R_{e}\right)$ only crosses the $R_{e}$-axis once. This crossing corresponds to the terms with exponents of $n+1$ or $m+1$ dominating the value of $P\left(R_{e}\right)$, resulting in a unique equilibrium point.

Fig. 2-3 plots the nullclines for a small and large value of $\alpha_{0}$, leading to a change in the number of equilibrium points from 3 to 1 , respectively. In the sequel, a sufficiently large $\alpha_{0}$ was used to ensure a unique equilibrium point.

By the Poincaré-Bendixson theorem, if the equilibrium point is unstable and not a saddle point, there exists a limit cycle. Since this system approximation is two 
dimensional, the eigenvalues of the Jacobian matrix and the condition for the existence of a limit cycle is given as (where $J$ is the Jacobian matrix, $T r$ is trace, and det is determinant):

$$
\begin{aligned}
& \lambda_{1,2}=\frac{\operatorname{Tr}(J) \pm \sqrt{\operatorname{Tr}(J)^{2}-4 \operatorname{det}(J)}}{2} \\
& \operatorname{Re}\left[\lambda_{1,2}\right]>0 \Longleftrightarrow \operatorname{Tr}(J)>0, \operatorname{det}(J)>0 .
\end{aligned}
$$

The Jacobian $J$ is given by: $J=\left.\left[\begin{array}{cc}\frac{\partial f}{\partial A} & \frac{\partial f}{\partial R} \\ \frac{\partial g}{\partial A} & \frac{\partial g}{\partial R}\end{array}\right]\right|_{\left(A_{e}, R_{e}\right)}$. The determinant of $J$ is given by (evaluated at the equilibrium point): $\operatorname{det}(J)=\frac{\partial f}{\partial A} \frac{\partial g}{\partial R}-\frac{\partial f}{\partial R} \frac{\partial g}{\partial A}$. Since $\frac{\partial f}{\partial A}, \frac{\partial g}{\partial A}>0$ and $\frac{\partial g}{\partial R}, \frac{\partial f}{\partial R}<0$, we have that $\frac{\partial f}{\partial A} \frac{\partial g}{\partial R}$ is always negative and $-\frac{\partial f}{\partial R} \frac{\partial g}{\partial A}$ is always positive. To see how $\operatorname{det}(J)>0$ can be graphically verified, we consider how these conditions translate in terms of the nullcline slopes at the equilibrium point. From (2.4)-(2.5), the nullclines satisfy $f(A, R)=0$ and $g(A, R)=0$. Let $X_{A}(A), X_{R}(A)$ be the locally unique solution to $f(A, R)=0$ and $g(A, R)=0$ about the equilibrium point, respectively, and so $R=X_{A}(A) \Longrightarrow f\left(A, X_{A}(A)\right)=0$ and $R=X_{R}(A) \Longrightarrow g\left(A, X_{R}(A)\right)=0$. The nullclines are therefore defined by $R=X_{A}(A)$ and $R=X_{R}(A)$. By the Implicit Function Theorem [29], $\frac{d X_{A}}{d A}=-\frac{(\partial f / \partial A)}{(\partial f / \partial R)}$ and $\frac{d X_{R}}{d A}=-\frac{(\partial g / \partial A)}{(\partial g / \partial R)}$ at the equilibrium point. Since $\frac{\partial f}{\partial R}<0$ and $\frac{\partial g}{\partial R}<0$ :

$$
\begin{aligned}
& \left.\frac{d X_{A}}{d A}\right|_{\left(A_{e}, R_{e}\right)}=\frac{\left.(\partial f / \partial A)\right|_{\left(A_{e}, R_{e}\right)}}{|(\partial f / \partial R)|_{\left(A_{e}, R_{e}\right)} \mid} \\
& \left.\frac{d X_{R}}{d A}\right|_{\left(A_{e}, R_{e}\right)}=\frac{\left.(\partial g / \partial A)\right|_{\left(A_{e}, R_{e}\right)}}{|(\partial g / \partial R)|_{\left(A_{e}, R_{e}\right)} \mid}
\end{aligned}
$$

$\operatorname{det}(J)>0$ requires (at the equilibrium point), $\frac{\partial g}{\partial A}\left|\frac{\partial f}{\partial R}\right|>\frac{\partial f}{\partial A}\left|\frac{\partial g}{\partial R}\right|$, that is, $\frac{\frac{\partial g}{\partial A}}{\left|\frac{\partial g}{\partial R}\right|}>\frac{\frac{\partial f}{\partial A}}{\left|\frac{\partial f}{\partial R}\right|}$ which, from (2.13)-(2.14), is equivalent to $\frac{d X_{R}}{d A}>\frac{d X_{A}}{d A}$. Therefore, the condition $\operatorname{det}(J)>0$ is guaranteed if the slope of the nullcline defined by $g=0$ is larger than the slope of the nullcline defined by $f=0$ at the equilibrium point. The four qualitatively different types of unique equilibrium points are given in Fig. 2-4. In 

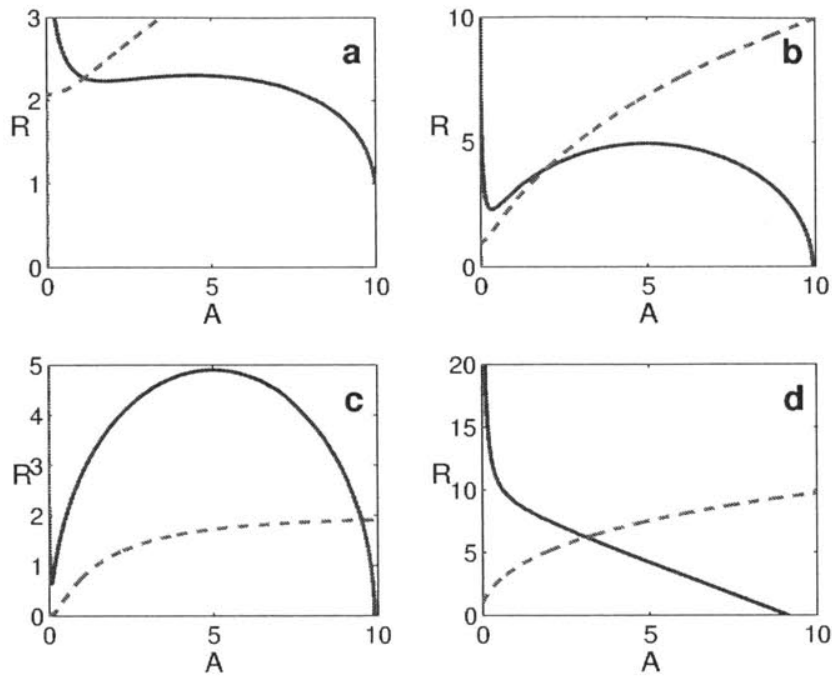

Figure 2-4: Four possible nullcline intersection regions of (2.4) and (2.5) given by blue solid $(f=0)$ and green dotted lines $(g=0)$, respectively. System parameters include $\alpha=20 \mathrm{nMhr}^{-1}, k_{A}=k_{R}=1 \mathrm{nM}, \alpha_{0}=40(\mathbf{a}), 2(\mathbf{b}), 0.1(\mathbf{c}), 2(\mathbf{d}) \mathrm{nMhr}^{-1}$, $\delta_{A}=\delta_{R}=1 \mathrm{hr}^{-1}, \kappa_{A}=\kappa_{R}=1 \mathrm{hr}^{-1}, \gamma_{A}=2 \mathrm{hr}^{-1}, \gamma_{R}=1(\mathbf{a b d}), 10(\mathbf{c}) \mathrm{hr}^{-1}, n=2(\mathbf{a b c})$ $1(\mathbf{d}), m=2(\mathbf{a b c}) 1(\mathbf{d})$.

Fig. 2-4a, 2-4c, 2-4d, the slope of $X_{A}(A)$ at the equilibrium point is negative. Given equality (2.13), this implies that $\frac{\partial f}{\partial A}<0$. Since $\operatorname{Tr}(J)=\frac{\partial f}{\partial A}+\frac{\partial g}{\partial R}$ and $\frac{\partial g}{\partial R}<0$, it follows that in these cases, the equilibrium point is stable. An unstable equilibrium point can occur only when the nullclines intersect as in Fig. 2-4b. We therefore focus on this case in the sequel.

To determine which parameter values can make the trace positive, we observe its analytical expression which is given by:

$$
\begin{aligned}
& \operatorname{Tr}(J)=\frac{\alpha \kappa_{A}}{\delta_{A}}\left(\frac{n\left(A_{e} / k_{A}\right)^{n-1}\left[1-\frac{\alpha_{0}}{\alpha}+\left(R_{e} / k_{R}\right)^{m}\right]}{k_{A}\left[1+\left(A_{e} / k_{A}\right)^{n}+\left(R_{e} / k_{R}\right)^{m}\right]^{2}}\right) \\
& -\gamma_{A}-\frac{\alpha \kappa_{R}}{\delta_{R}}\left(\frac{m\left(R_{e} / k_{R}\right)^{m-1}\left(\left(A_{e} / k_{A}\right)^{n}+\frac{\alpha_{0}}{\alpha}\right)}{k_{R}\left[1+\left(A_{e} / k_{A}\right)^{n}+\left(R_{e} / k_{R}\right)^{m}\right]^{2}}\right)-\gamma_{R} .
\end{aligned}
$$

The last three terms are always negative. The expression for the trace suggests that it can be made positive by increasing $\frac{\kappa_{A} \alpha}{\delta_{A}}$. This ratio is the maximum production rate of $\mathrm{A}$, which corresponds to having a fully active promoter (i.e., $A \rightarrow \infty$ ) leading to a steady-state $m_{A}$ value of $\frac{\alpha}{\delta_{A}}$. Increasing $\frac{\kappa_{A} \alpha}{\delta_{A}}$, however, does not guarantee that the 


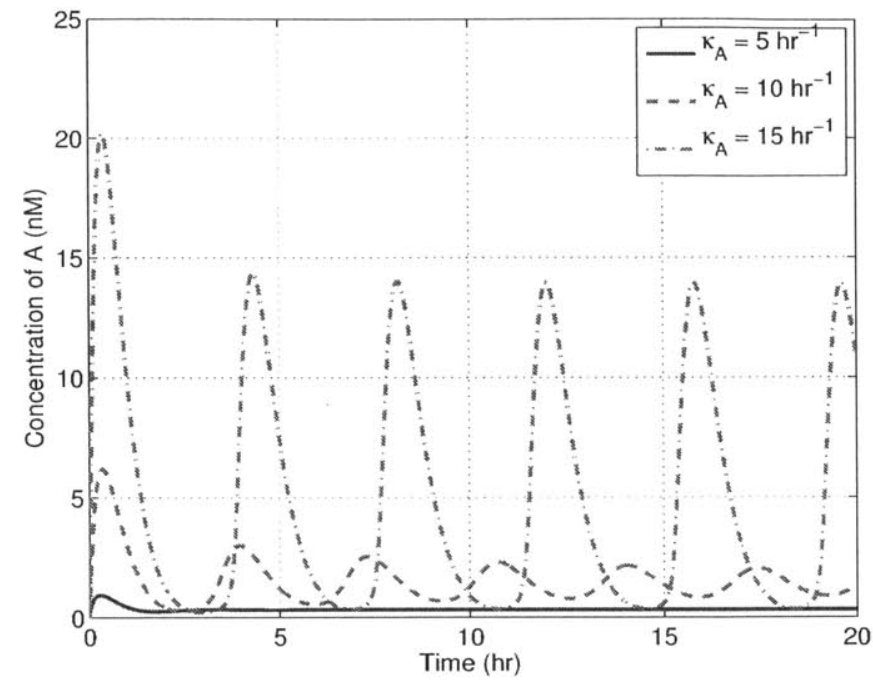

Figure 2-5: Change in response of A from decaying to stable oscillations as $\kappa_{A}$ increases for system given by (2.4)-(2.5). System parameters include $\alpha=1200 \mathrm{hr}^{-1} /$ gene, $k_{A}=k_{R}=1 \mathrm{nM}, \alpha_{0}=120 \mathrm{hr}^{-1} /$ gene, $\delta_{A}=\delta_{R}=10 \mathrm{hr}^{-1}, \kappa_{R}=50 \mathrm{hr}^{-1}, \gamma_{A}=3.3 \mathrm{hr}^{-1}$, $\gamma_{R}=1 \mathrm{hr}^{-1}, \mathrm{n}=2, \mathrm{~m}=2$.

two-state system approximation exhibits sustained oscillations, since $\alpha, \kappa_{A}, \delta_{A}$ affect the value of the equilibrium point, making it difficult to identify their effect on the trace. Furthermore, increasing $\alpha$ would also increase the third term. Nevertheless, Fig. 2-5 demonstrates that increasing $\kappa_{A}$ does lead to oscillations. For a stable limit cycle, $\alpha$ was set an order of magnitude larger than $\alpha_{0}$ (corresponding to Fig. 2-4b) and $\kappa_{A}$ was set sufficiently large to ensure a positive trace. Relatively large values of $\alpha_{0}$ were found to make the equilibrium point stable (i.e., for $\alpha_{0} \approx \alpha$ as in Fig. 2-4a).

To summarize our findings, the system given by (2.4)-(2.5) has a unique equilibrium point for sufficiently large $\alpha_{0}$. The conditions for an unstable equilibrium point include (i) the nullclines need to intersect with positive slope at the equilibrium point ( $\alpha_{0}$ should not be too large), (ii) the slope of the nullcline defined by $g=0$ should be greater than the slope of the nullcline of $f=0$ at the equilibrium point, and (iii) sufficiently large maximum production rate of $\mathrm{A}\left(\kappa_{A}\right)$ compared to that of $\mathrm{R}$. 


\section{Chapter 3}

\section{Oscillator with downstream load}

\subsection{Downstream load to A}

Consider A transcriptionally regulating downstream promoter sites represented schematically in Fig. 3-1.

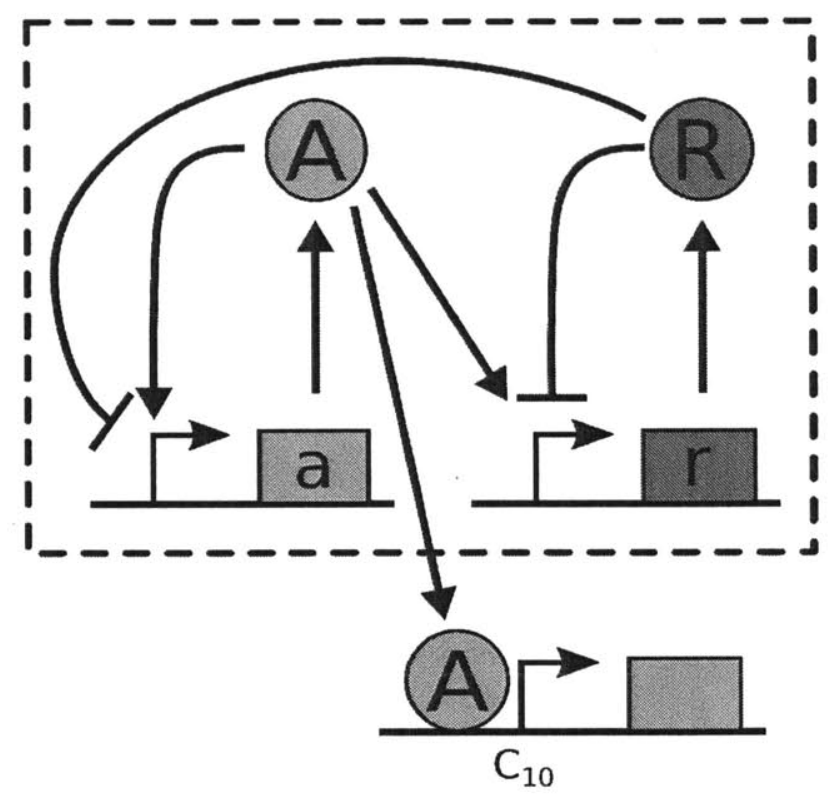

Figure 3-1: Load to A by downstream promoter sites $C_{10}$. The original two-state oscillator system is given in the dashed box.

This transcriptional regulation occurs by A binding to DNA promoter sites. Let the free promoter sites be denoted as $C_{10}$ and the sites bound to $\mathrm{A}$ be denoted as $C_{11}$. Since DNA does not decay, the total concentration of promoter sites is conserved, 
that is $C_{10}+C_{11}=C_{t 1}$, where $C_{t 1}$ represents the total concentration of the free and bound promoter sites. The complex formation reaction is given by: $C_{10}+A \underset{d}{\stackrel{a}{\rightleftarrows}} C_{11}$. The dynamics of A change in the new three-state system equations, which are now given by:

$$
\begin{aligned}
\dot{A} & =\frac{\kappa_{A}}{\delta_{A}} \frac{\alpha\left(A / k_{A}\right)^{n}+\alpha_{0}}{1+\left(A / k_{A}\right)^{n}+\left(R / k_{R}\right)^{m}}-\gamma_{A} A-\dot{C}_{11}, \\
\dot{R} & =\frac{\kappa_{R}}{\delta_{R}} \frac{\alpha\left(A / k_{A}\right)^{n}+\alpha_{0}}{1+\left(A / k_{A}\right)^{n}+\left(R / k_{R}\right)^{m}}-\gamma_{R} R, \\
\dot{C}_{11} & =a\left(C_{t 1}-C_{11}\right) A-d C_{11} .
\end{aligned}
$$

Increased loading to $A$ (increased $C_{t 1}$ ) decreases the amplitude of oscillations to the point of quenching oscillations as shown in Fig. 3-2. The response of $R$ is qualitatively similar to that of $A$ : higher $C_{t 1}$ values cause smaller amplitude oscillations until the clock is quenched.

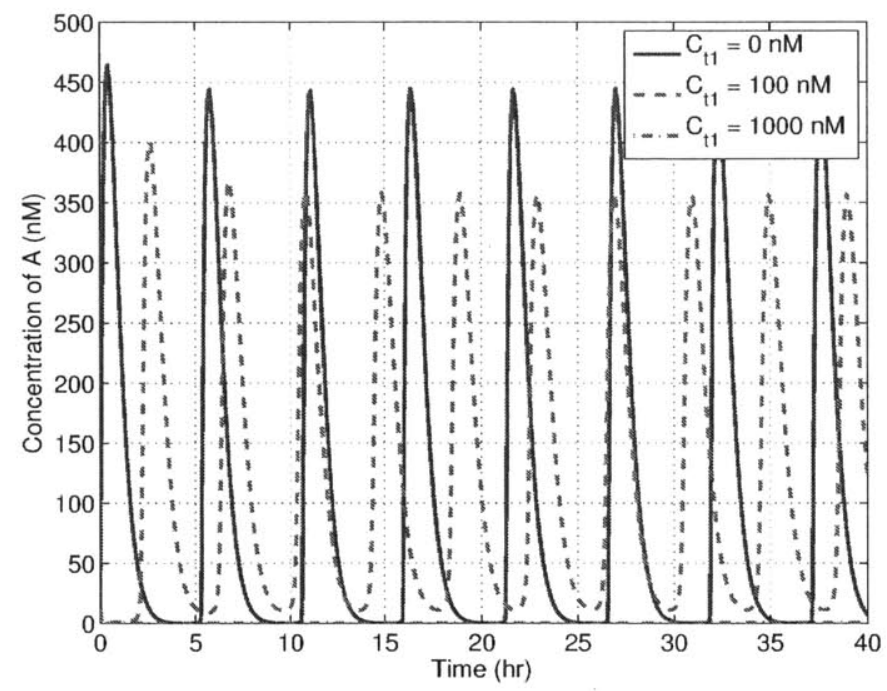

Figure 3-2: Simulation of (3.1) with system parameters: $\alpha=1200 \mathrm{hr}^{-1} /$ gene, $k_{A}=$ $k_{R}=1 \mathrm{nM}, \alpha_{0}=120 \mathrm{hr}^{-1} /$ gene, $\delta_{A}=\delta_{R}=10 \mathrm{hr}^{-1}, \kappa_{A}=\kappa_{R}=50 \mathrm{hr}^{-1}, \gamma_{A}=3.5 \mathrm{hr}^{-1}$, $\gamma_{R}=1 \mathrm{hr}^{-1}, \mathrm{n}=2, \mathrm{~m}=2$.

To simplify analysis using a reduced order model through singular perturbation, we follow the procedure in [23] to determine the retroactivity matrices of the system 
and as shown in Appendix B, we obtain (3.2).

$$
\begin{aligned}
& \dot{A}=\frac{\left(1+\frac{A}{K_{d 1}}\right)^{2}}{\left(1+\frac{A}{K_{d 1}}\right)^{2}+\frac{C_{t 1}}{K_{d 1}}}\left(\frac{\kappa_{A}}{\delta_{A}} \frac{\alpha\left(\frac{A}{k_{A}}\right)^{n}+\alpha_{0}}{1+\left(\frac{A}{k_{A}}\right)^{n}+\left(\frac{R}{k_{R}}\right)^{m}}-\gamma_{A} A\right), \\
& \dot{R}=\frac{\kappa_{R}}{\delta_{R}} \frac{\alpha\left(A / k_{A}\right)^{n}+\alpha_{0}}{1+\left(A / k_{A}\right)^{n}+\left(R / k_{R}\right)^{m}}-\gamma_{R} R \text {. }
\end{aligned}
$$

Note that when there is no load (i.e., $C_{t 1}=0$ ), we recover $(2.4)-(2.5)$. The dynamics of $\mathrm{R}$ have not changed from (2.5). The new $\dot{A}$ equation is the product of a loading term (always positive and less than 1) and (2.4) and so the dynamics of A are effectively slower due to the load. The new system nullclines are identical to that of (2.4)-(2.5), since the nullcline defined by $\dot{A}=0$ is independent of the loading term. Therefore, the equilibrium point $\left(A_{e}, R_{e}\right)$ for the unloaded two-state system and for the loaded reduced model system is the same.

To analytically investigate what the effect of the load is on the clock's behavior, we analyze the stability of the equilibrium point for the two-state system (3.2). Since the sign of $\operatorname{det}(J)$ is not affected by the presence of the load, as before we can guarantee that $\operatorname{det}(J)>0$ by requesting that the slope of the nullcline defined by $\dot{R}=0$ is greater than the slope of the nullcline defined by $\dot{A}=0$ at the equilibrium point. If this is satisfied, the real part of the eigenvalues of the reduced system can be made positive if $\operatorname{Tr}(J)>0$, which is given by:

$\operatorname{Tr}(J)=\frac{\left(\frac{\kappa_{A}}{\delta_{A}}\left(\frac{n\left(\frac{A_{e}}{k_{A}}\right)^{n-1}\left(\alpha+\alpha\left(\frac{R_{e}}{k_{R}}\right)^{m}-\alpha_{0}\right)}{k_{A}\left[1+\left(\frac{A_{e}}{k_{A}}\right)^{n}+\left(\frac{R_{e}}{k_{R}}\right)^{m}\right]^{2}}\right)-\gamma_{A}\right)}{1+\frac{C_{t 1} / K_{d 1}}{\left(1+A_{e} / K_{d 1}\right)^{2}}}-\frac{\kappa_{R}}{\delta_{R}}\left(\frac{m\left(\frac{R_{e}}{k_{R}}\right)^{m-1}\left(\alpha\left(\frac{A_{e}}{k_{A}}\right)^{n}+\alpha_{0}\right)}{k_{R}\left[1+\left(\frac{A_{e}}{k_{A}}\right)^{n}+\left(\frac{R_{e}}{k_{R}}\right)^{m}\right]^{2}}\right)-\gamma_{R}$

The last two terms in the expression for the trace are negative and independent of $C_{t 1}$. As $C_{t 1}$ becomes larger, the magnitude of the first term decreases. Therefore, even if $\operatorname{Tr}(J)$ is initially positive when $C_{t 1}=0$, for large enough $C_{t 1}$ the expression in the last line dominates and $\operatorname{Tr}(J)<0$. Therefore, for sufficiently large load to A, the system will not exhibit a limit cycle and will converge to the stable equilibrium point. 


\subsection{Downstream load to $\mathrm{R}$}

Now consider the case when only $\mathrm{R}$ has downstream load (no $C_{t 1}$ ) represented schematically in Fig. 3-3.

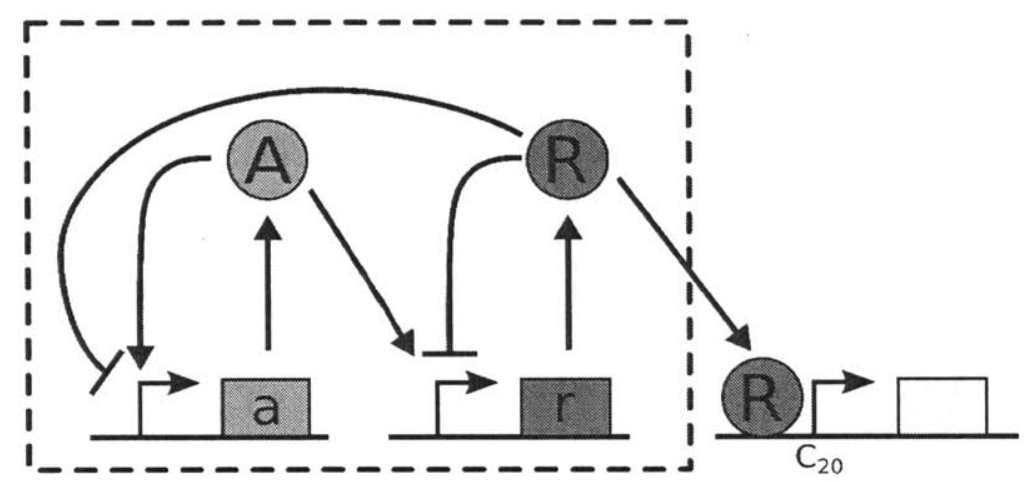

Figure 3-3: Load to $\mathrm{R}$ by downstream promoter sites $C_{20}$. The original two-state oscillator system is given in the dashed box.

Let the free promoter sites be denoted as $\mathrm{C}_{20}$ and the sites bound to $\mathrm{R}$ be denoted as $\mathrm{C}_{21}$. Once again, since DNA does not decay, the total concentration of the promoter sites is conserved and is given by $C_{20}+C_{21}=C_{t 2}$. The complex formation reaction is given by: $C_{20}+R \underset{d^{\prime}}{\stackrel{a^{\prime}}{\rightleftarrows}} C_{21}$. The dynamics of $\mathrm{R}$ have changed and the three-state system equations are given by:

$$
\begin{aligned}
\dot{A} & =\frac{\kappa_{A}}{\delta_{A}} \frac{\alpha\left(A / k_{A}\right)^{n}+\alpha_{0}}{1+\left(A / k_{A}\right)^{n}+\left(R / k_{R}\right)^{m}}-\gamma_{A} A, \\
\dot{R} & =\frac{\kappa_{R}}{\delta_{R}} \frac{\alpha\left(A / k_{A}\right)^{n}+\alpha_{0}}{1+\left(A / k_{A}\right)^{n}+\left(R / k_{R}\right)^{m}}-\gamma_{R} R-\dot{C}_{21}, \\
\dot{C}_{21} & =a^{\prime}\left(C_{t 2}-C_{21}\right) R-d^{\prime} C_{21} .
\end{aligned}
$$

An initially quenched oscillator can be brought back to functioning by loading $\mathrm{R}$ with large enough promoter concentration. To demonstrate this "activation" of the gene oscillator, consider the case when the real part of the linearized system eigenvalues are negative, but close to the origin. Fig. 3-4 demonstrates the stable system transitioning to limit cycle behavior after $t=100 \mathrm{hr}$ by the addition of a downstream system to $\mathrm{R}$ (with $C_{t 2}=1000 \mathrm{nM}$ ).

To understand why this occurs, once again we consider how the eigenvalues of 


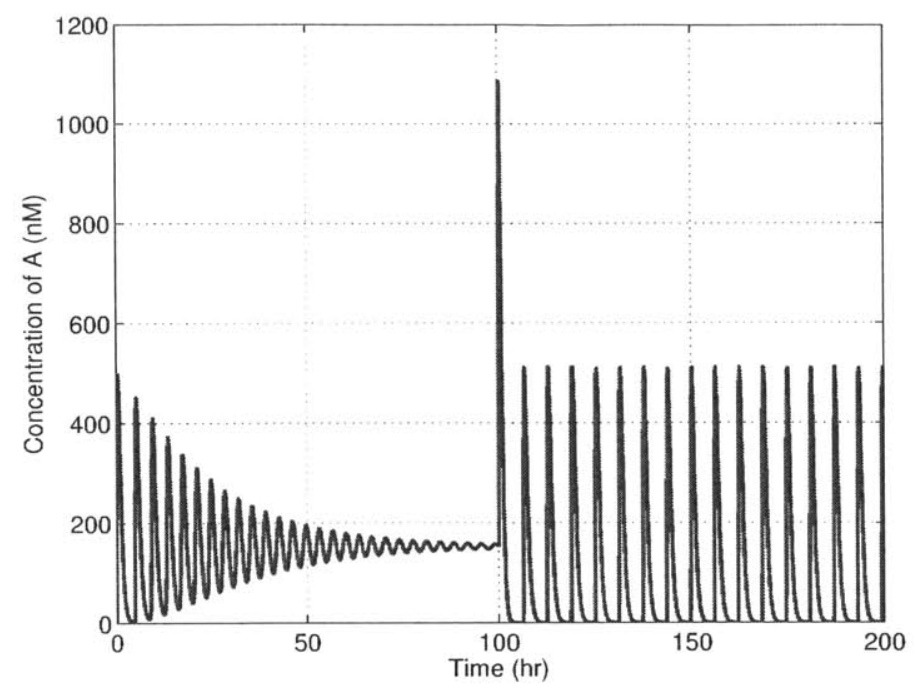

Figure 3-4: Simulation of (3.3) demonstrating the transition from decayed response to oscillatory behavior by addition of downstream $C_{t 2}=1000 \mathrm{nM}$ at $t=100 \mathrm{hr}$. System parameters include: $\alpha=1200 \mathrm{hr}^{-1} /$ gene, $k_{A}=k_{R}=1 \mathrm{nM}, \alpha_{0}=120 \mathrm{hr}^{-1} /$ gene, $\delta_{A}=\delta_{R}=10 \mathrm{hr}^{-1}, \kappa_{A}=\kappa_{R}=50 \mathrm{hr}^{-1}, \gamma_{A}=3.3 \mathrm{hr}^{-1}, \gamma_{R}=1 \mathrm{hr}^{-1}, \mathrm{n}=2, \mathrm{~m}=2$.

the linearized system change due to the addition of $C_{t 2}$ by assuming that complex formation $\left(\mathrm{C}_{21}\right)$ occurs significantly quicker than protein $(\mathrm{A}, \mathrm{R})$ dynamics for model reduction. By using singular perturbation once again, the system can be reduced to two-states:

$$
\begin{aligned}
\dot{A} & =\frac{\kappa_{A}}{\delta_{A}} \frac{\alpha\left(A / k_{A}\right)^{n}+\alpha_{0}}{1+\left(A / k_{A}\right)^{n}+\left(R / k_{R}\right)^{m}}-\gamma_{A} A \\
\dot{R} & =\frac{\left(1+\frac{R}{K_{d 2}}\right)^{2}}{\left(1+\frac{R}{K_{d 2}}\right)^{2}+\frac{C_{t 2}}{K_{d 2}}}\left(\frac{\kappa_{R}}{\delta_{R}} \frac{\alpha\left(\frac{A}{k_{A}}\right)^{n}+\alpha_{0}}{1+\left(\frac{A}{k_{A}}\right)^{n}+\left(\frac{R}{k_{R}}\right)^{m}}-\gamma_{R} R\right) .
\end{aligned}
$$

As before, $\operatorname{det}(J)>0$ can be verified graphically by ensuring that the slope of the nullcline defined by $\dot{R}=0$ is greater than that of the nullcline defined by $\dot{A}=0$ at the equilibrium point. Furthermore, the equilibrium point remains the same since the loading term to $\dot{R}$ is always positive. The expression for the trace of the linearized system with downstream load to $R$ is given by:

$\operatorname{Tr}(J)=\frac{\kappa_{A} \alpha}{\delta_{A}}\left(\frac{n\left(\frac{A_{e}}{k_{A}}\right)^{n-1}\left[1-\frac{\alpha_{0}}{\alpha}+\left(\frac{R_{e}}{k_{R}}\right)^{m}\right]}{k_{A}\left[1+\left(\frac{A_{e}}{k_{A}}\right)^{n}+\left(\frac{R_{e}}{k_{R}}\right)^{m}\right]^{2}}\right)-\gamma_{A}-\frac{\frac{\kappa_{R}}{\delta_{R}}\left(\frac{m\left(R_{e} / k_{R}\right)^{m-1}\left(\alpha\left(A_{e} / k_{A}\right)^{n}+\alpha_{0}\right)}{k_{R}\left[1+\left(\frac{A_{e}}{k_{A}}\right)^{n}+\left(\frac{R_{e}}{k_{R}}\right)^{m}\right]^{2}}\right)-\gamma_{R}}{1+\frac{C_{t 2} / K_{d 2}}{\left(1+\frac{R_{e}}{K_{d 2}}\right)^{2}}}$. 
The first term is always positive due to the assumption that the contribution to transcription due to leakiness is significantly less than that due to $\mathrm{A}_{n}: \mathrm{DNA}^{\mathrm{P}}$ (i.e., $\left.\frac{\alpha_{0}}{\alpha}<<1\right)$. For sufficiently large $\frac{\kappa_{A} \alpha}{\delta_{A}}$, the first term is larger in magnitude than $\gamma_{A}$. The last term is always negative, but as $C_{t 2}$ increases, its magnitude decreases. Therefore, if the value of the trace for the two-state reduced model system with load to $\mathrm{R}$ is initially negative (system trajectories converge to an equilibrium point), we would expect it to become positive for sufficiently large $C_{t 2}$, leading to linearized system eigenvalues with positive real part and limit cycle behavior. 


\section{Chapter 4}

\section{Oscillator with downstream load and}

\section{decay}

\subsection{Complex decay to A}

In the previous sections, we assumed that transcription factors were protected from degradation when bound to DNA. This is often a good assumption, which has also been experimentally validated for some transcription factors [32]. In other cases, however, a transcription factor can still be degraded when bound to DNA [1]. Here, we consider A transcriptionally regulating downstream promoter sites that decay at a constant rate when bound with A. The modified load reactions to A are given by:

$$
\begin{array}{r}
A+C_{10} \underset{d}{\stackrel{a}{\rightleftarrows}} C_{11}, \\
C_{11} \stackrel{\pi_{A}}{\rightarrow} C_{10} .
\end{array}
$$

The total concentration of the promoter sites is conserved as before, but the dynamics of the three-state system have changed to $\left(f_{1}(A, R)=\frac{\kappa_{A}}{\delta_{A}} \frac{\alpha\left(A / k_{A}\right)^{n}+\alpha_{0}}{1+\left(A / k_{A}\right)^{n}+\left(R / k_{R}\right)^{m}}\right.$, 
$\left.f_{2}(A, R)=\frac{\kappa_{R}}{\delta_{R}} \frac{\alpha\left(A / k_{A}\right)^{n}+\alpha_{0}}{1+\left(A / k_{A}\right)^{n}+\left(R / k_{R}\right)^{m}}\right):$

$$
\begin{aligned}
\dot{A} & =f_{1}(A, R)-\gamma_{A} A+d C_{11}-a A\left(C_{t 1}-C_{11}\right), \\
\dot{R} & =f_{2}(A, R)-\gamma_{R} R, \\
\dot{C}_{11} & =a A\left(C_{t 1}-C_{11}\right)-\left(d+\pi_{A}\right) C_{11} .
\end{aligned}
$$

To understand how the dynamics of the system are affected by the decay, we reduce the system to two-states by employing singular perturbation (see Appendix $\mathbf{C}$ for details), which results in the following $A$ dynamics $\left(p_{A}=\frac{d}{d+\pi_{A}}, K_{d 1}=\frac{d}{a}\right)$ :

$$
\dot{A}=\frac{f_{1}(A, R)-\gamma_{A} A-\frac{\pi_{A} p_{A} C_{t 1} A}{K_{d 1}+p_{A} A}}{1+\frac{p_{A} K_{d 1} C_{t 1}}{\left(K_{d 1}+p_{A} A\right)^{2}}}
$$

The $\dot{A}$ equation is now more difficult to analyze than (3.2), so we employ standard software bifurcation tools [33] to observe changes to system stability (Fig. 4-1). Both systems (load to A with and without complex decay) and are initialized with an unstable equilibrium point (represented by the dashed line). As load is changed, the equilibrium value (both $A_{e}$ and $R_{e}$ ) of the system with complex decay decreases. This is due to permanent sequestration of $\mathrm{A}$ by the complex decay process, leading to a smaller $A_{e}$ as $C_{t 1}$ is increased. Since A transcriptionally activates R, a lower concentration of $\mathrm{A}$ causes a lower $R_{e}$. Complex decay also greatly diminishes the system's robustness to downstream load. Both systems exhibit a subcritical Hopf bifurcation, wherein a pair of complex conjugate eigenvalues of the linearized system crosses the imaginary plane from right to left. For values of $C_{t 1}$ larger than the load corresponding to the bifurcation point, both systems will converge to their respective stable equilibrium point and there will be no stable oscillations. However, for realistic parameter values, the system with no load exhibits oscillations up to $200 \mathrm{nM}$ of load to A compared to just $16 \mathrm{nM}$ when $C_{11}$ can decay. 


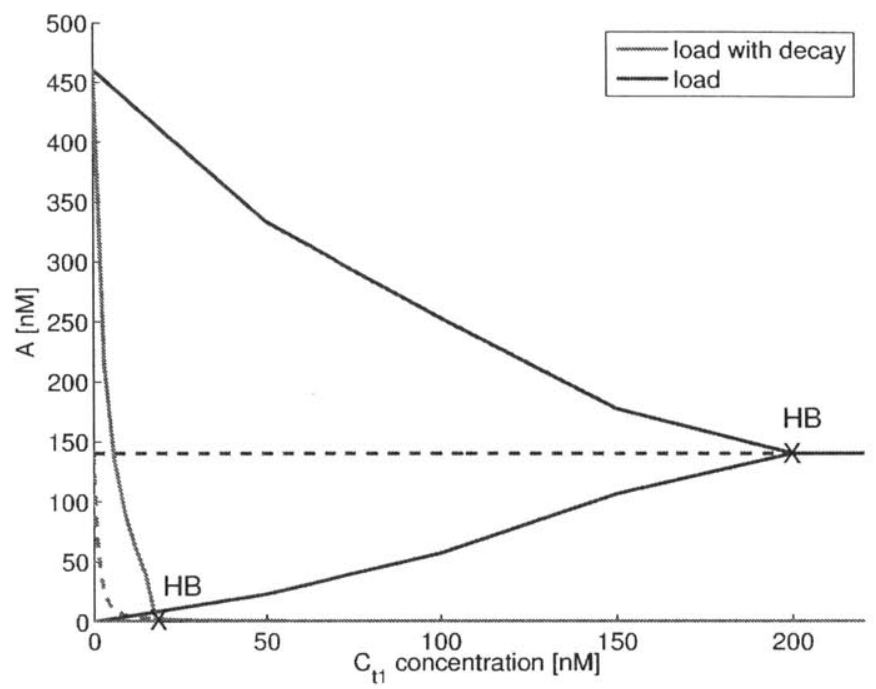

Figure 4-1: Bifurcation diagram of system described by (4.2). As the bifurcation parameter, $C_{t 1}$ is increased, both systems undergo a subcritical Hopf bifurcation. System parameters include $\alpha=1200 \mathrm{hr}^{-1} /$ gene, $k_{A}=k_{R}=1 \mathrm{nM}, \alpha_{0}=120 \mathrm{hr}^{-1} /$ gene, $\delta_{A}=\delta_{R}=10 \mathrm{hr}^{-1}, \kappa_{A}=\kappa_{R}=50 \mathrm{hr}^{-1}, \gamma_{A}=3.4 \mathrm{hr}^{-1}, \gamma_{R}=1 \mathrm{hr}^{-1}, \mathrm{n}=2, \mathrm{~m}=2, K_{d 1}=1$, $\pi_{A}=1 \mathrm{hr}^{-1}$.

\subsection{Complex decay to $\mathrm{R}$}

We repeat the procedure for $\mathrm{R}$ transcriptionally regulating a downstream system with complex decay given by:

$$
\begin{array}{r}
R+C_{20} \underset{d^{\prime}}{\stackrel{a^{\prime}}{\rightleftarrows}} C_{21}, \\
C_{21} \stackrel{\pi_{R}}{\rightarrow} C_{20} .
\end{array}
$$

Singular perturbation is once again used, leading to $\left(p_{R}=\frac{d^{\prime}}{\pi_{R}+d^{\prime}}, K_{d 2}=\frac{d^{\prime}}{a^{\prime}}\right)$ :

$$
\dot{R}=\frac{f_{2}(A, R)-\gamma_{R} R-\frac{\pi_{R} p_{R} C_{t 2} R}{K_{d 2}+p_{R} R}}{1+\frac{p_{R} K_{d 2} C_{t 2}}{\left(K_{d 2}+p_{R} R\right)^{2}}} .
$$

Both systems are initialized with a stable equilibrium point in Fig. 4-2. The bifurcation diagram demonstrates that the system without complex decay undergoes a supercritical Hopf bifurcation (i.e., makes the transition from a stable to unstable equilibrium point). For a large range of load (0-1000 nM) and complex decay rates 


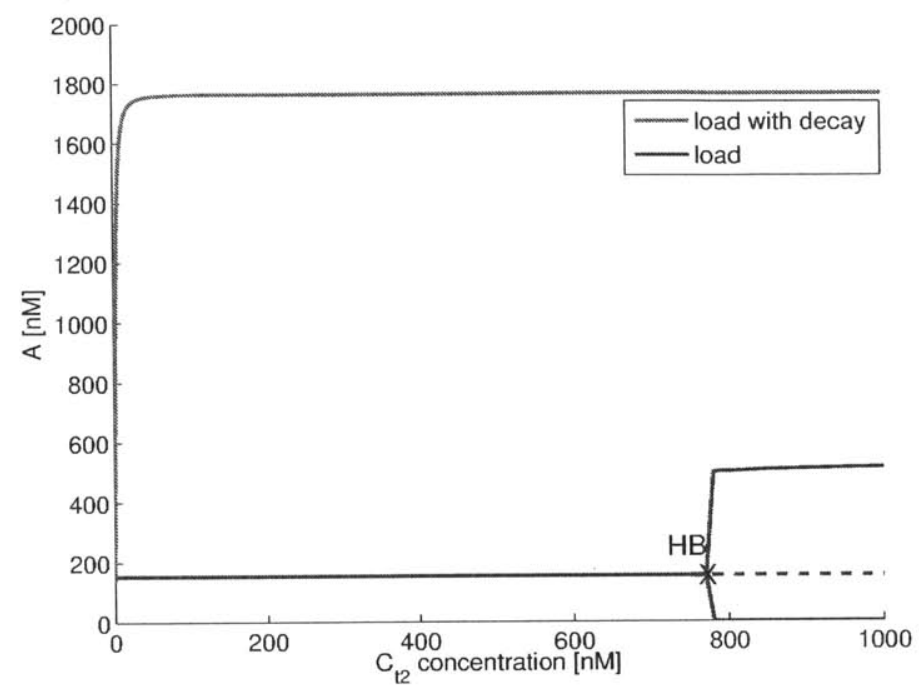

Figure 4-2: Bifurcation diagram of system described by (4.3). As the bifurcation parameter, $C_{t 2}$, is increased, the system with only load undergoes a supercritical Hopf bifurcation while the system with decay is always stable. System parameters include $\alpha=1200 \mathrm{hr}^{-1} /$ gene, $k_{A}=k_{R}=1 \mathrm{nM}, \alpha_{0}=120 \mathrm{hr}^{-1} /$ gene, $\delta_{A}=\delta_{R}=10 \mathrm{hr}^{-1}$, $\kappa_{A}=\kappa_{R}=50 \mathrm{hr}^{-1}, \gamma_{A}=3.3 \mathrm{hr}^{-1}, \gamma_{R}=1 \mathrm{hr}^{-1}, \mathrm{n}=2, \mathrm{~m}=2, K_{d 2}=1, \pi_{R}=1 \mathrm{hr}^{-1}$.

$\left(\pi_{R}=0.5-10 \mathrm{hr}^{-1}\right)$, the system with complex decay does not exhibit oscillations. The decay causes the steady-state value of A to increase, but $R_{e}$ decreases after sufficiently large $C_{t 2}$ (not shown in Fig. 4-2). In the former case of complex decay with load on A, the permanent sequestration of A resulted in a decrease of both the steady state value of $\mathrm{A}$ and $\mathrm{R}$, since $\mathrm{A}$ activates itself and $\mathrm{R}$. Here, the decay of $\mathrm{R}$ enhances the production of A (due to weakened repression), which in turn increases production of $\mathrm{R}$. This trade-off is dominated by A activating $\mathrm{R}$ for small values of load and by the load and decay for larger values of $C_{t 2}$, causing an eventual decrease in $R_{e}$. 


\section{Chapter 5}

\section{Experimental Protocol Development}

\subsection{Introduction}

To verify the effects of load from the model, an experimental setup needed to be developed for imaging cells. Commercial imaging platforms, such as cellASIC ONIX, provide the necessary features, but we did not have access to such systems and are expensive. This section discusses a "home-made" substitute and details a cost-effective setup consisting of equipment and instruments that were easily acquired and/or already available in our lab.

The basic steps involved in experimental validation begin with obtaining a strain (or cells) that will "host" the oscillator (in this case, a form of E. coli). The next step is to transform the strain with the plasmids that contain the oscillator (i.e., inserting the plasmids in the host cell). Plasmids are circular DNA molecules that replicate independently from the host cell's chromosomal DNA and can be engineered to have particular genes, which after transcription and translation produce protein. Two plasmids (detailed below) contain the three genes that compromise the oscillator: araC (activator), lacI (repressor), and yem-GFP (green fluorescent protein, or reporter). The reporter gene has the same promoter (region of DNA that initiates transcription of a particular gene) as the activator and repressor, and so the GFP concentration (observed using a fluorescence microscope) tracks the behavior of the oscillator. 

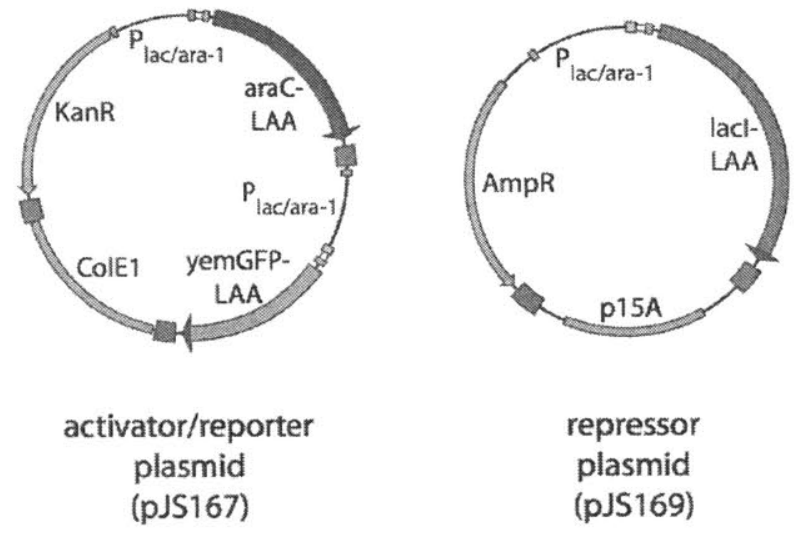

Figure 5-1: Plasmids pJS167 and pJS169 that contain the activator-repressor oscillator. Image obtained from [9].

\section{$5.2 \quad$ Plasmid and strain}

The E. coli strain (JS006) used for experimentation was gifted to us by the Biodynamics Laboratory at the University of California, San Diego (Prof. Jeff Hasty's group). ara $C$ and $l a c I$, which correspond to the activator and repressor genes, respectively, were deleted from the strain to ensure that only the $\operatorname{araC}$ and $l a c I$ genes on the plasmids produced the activator and repressor protein, respectively. The genetic oscillator is contained in two plasmids, pJS167 and pJS169, as shown in Fig. 5-1. Plasmids, in general, have 3 key components: the origin of replication (ORI), antibiotic resistant gene, and inserted/target genes. The ORI is the site of DNA replication (ColE1 in pJS167 and p15A in pJS169) and determines the number of plasmid copies that will persist in each cell. The antibiotic resistant gene (Kanamycin resistance in pJS167 and Ampicillin resistance in pJS169) is a selection marker; antibiotics are added to cell cultures to ensure that only the desired cells (i.e., those with the plasmids with the antibiotic resistant gene) can grow and replicate. The target gene(s) are the genes of interest and in this case, make up the oscillator (i.e., araC and yem-GFP in pJS167 and lacI in pJS169). A schematic of the steps involved in building the oscillator is given in Fig. 5-2. 


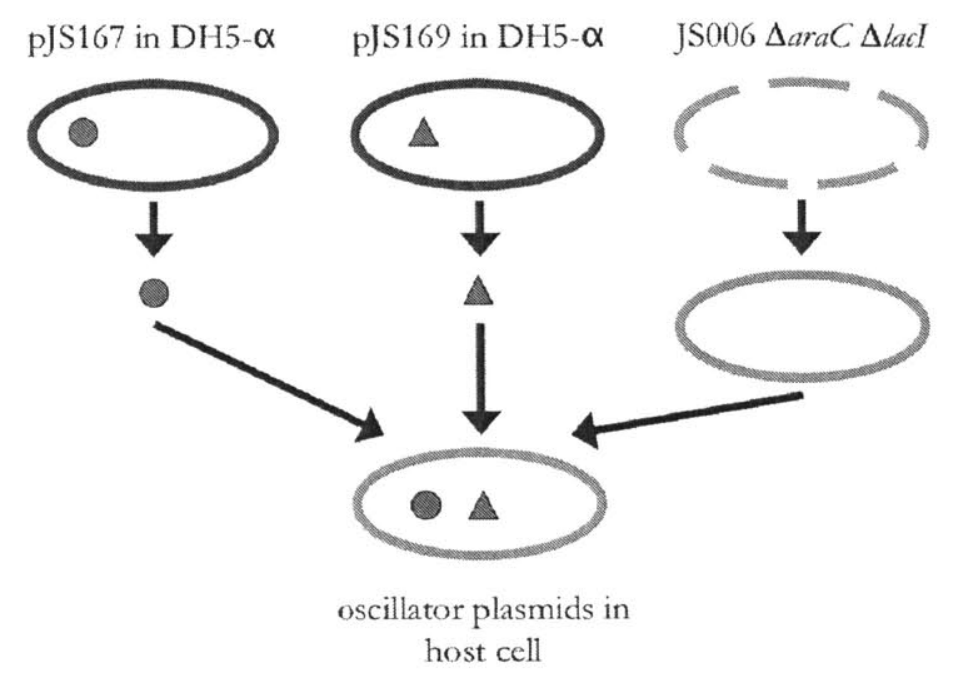

Figure 5-2: Schematic of oscillator circuit construction. The plasmids, pJS167 (red circle) and pJS169 (green triangle), are originally in the DH5- $\alpha$ strain (blue oval) and are purified using the Zymo Research ZR Plasmid Miniprep Kit. The host cell (dashed orange oval) is made competent and transformed (see Appendix D for protocol) with the extracted plasmids to yield the oscillator circuit.

\subsection{Trapping cells}

We experimented with 3 set-ups to determine which one could best trap cells for imaging. The first one involved coating a perfusion chamber with Concanavalin A (ConA), a carbohydrate-binding protein that traps E. coli. The second protocol used an agarose gel, that traps cells on its top layer as it solidifies. The last protocol (currently in use) involves microfluidics and a printed circuit board (PCB) heater. The following describes in the detail the protocol for each of the three methods.

\subsubsection{ConA protocol}

Sticker-based perfusion chamber strips manufactured by Grace Bio-Labs are silicone chambers bonded to transparent plastic stickers (with dimensions $3 \mathrm{~mm} \times 32 \mathrm{~mm} \times$ $0.6 \mathrm{~mm}$ and $55 \mu \mathrm{L}$ volume). They consist of an inlet and output port, which is used to load cells and media. The chamber is adhered to a cover glass using Loctite super glue. A solution of $1 \mathrm{~g} / \mathrm{mL}$ ConA is pipetted into the chamber to treat the chamber walls for $30 \mathrm{~min}$. After flushing the ConA and cleaning the chamber with sterilized 

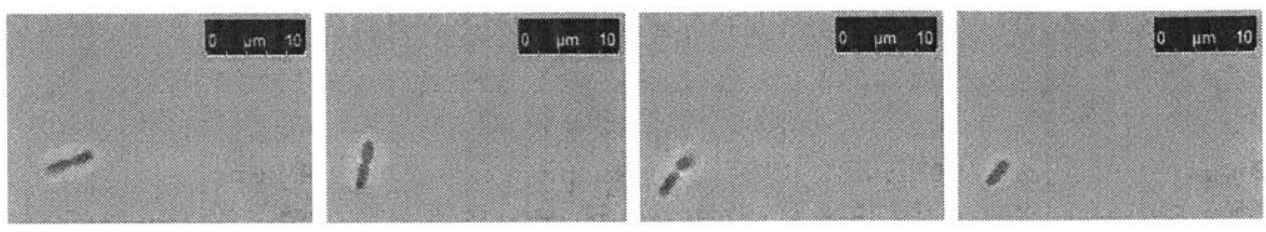

Figure 5-3: Time-lapse bright-field microscopy using ConA protocol. Interval between images is $12 \mathrm{~min}$. After replication (third frame), the daughter cell is not constrained by the chamber wall and disappears from the frame of view.

water, the chamber is loaded with the cell culture suspension for $10 \mathrm{~min}$ (to allow the cells to be affixed on the ConA coated chamber walls). The free cells are then flushed from the chamber by pipetting with sterilized water. Tubing is then fitted to the inlet and outlet ports and sealed using epoxy. The outlet tube empties to a biological waste container, while the inlet tube runs through an inline solution heater (Warner Instruments SF-28) and is connected to a syringe, containing LB media, selection markers (1 $\mu \mathrm{g} / \mathrm{mL}$ Ampicillin, $1 \mu \mathrm{g} / \mathrm{mL}$ Kanamycin), and inducers (2 mM IPTG, $0.7 \%$ arabinose). Flow rate is controlled by a pump (Harvard Apparatus Pump 11 Elite) and is set to $55 \mu \mathrm{L} / \mathrm{hr}$ (i.e., to replenish media in the chamber every hour). The chamber itself is positioned on top of a heater stand. Both heaters (solution heater and heater stand) are controlled by a dual channel heater controller (Warner Instruments TC-344B) and are set to operate at $37^{\circ} \mathrm{C}$. The fixed cells were monitored under both bright-field (white light) and fluorescence microscopy for at least 5 hrs.

It was observed that most of the cells were not firmly stuck to the chamber walls and tended to swim away from the frame of view within a few minutes. Few cells were adhered to the wall on one end but the movement of the free end made focusing difficult. We would sometimes get lucky and be able to image a cell stuck to the chamber wall on both ends but after it replicated, the daughter cell typically swam away (see Fig. 5-3). These results did not change even after using a $10 \mathrm{~g} / \mathrm{mL}$ ConA solution to coat the chamber wall or after loading the chamber with a mixture of the cell culture suspension and ConA (to increase cell-ConA exposure time). 


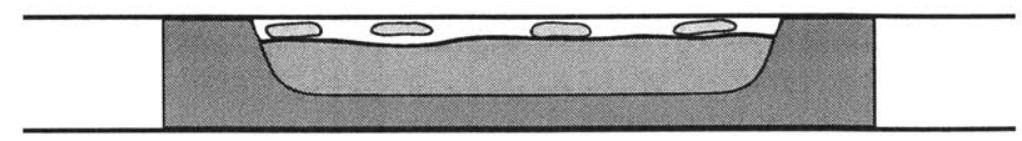

Figure 5-4: Side view of experiment setup with the agarose pad protocol. The cover glass (black lines) are placed on either side of the perfusion chamber (orange), which is filled with the agar gel (light pink). The cells (green) are sandwiched between the top layer of the gel and the top cover glass. This diagram intentionally exaggerates the space between the agar gel and the top cover glass to demonstrate that there is sufficient space for the cells to traverse despite firmly attaching the cover glass to the chamber.

\subsubsection{Agarose pad protocol}

A $1 \mathrm{~mL}$ solution of LB, selection markers $(1 \mu \mathrm{g} / \mathrm{mL}$ Ampicillin, $1 \mu \mathrm{g} / \mathrm{mL}$ Kanamycin $)$, and inducers ( $2 \mathrm{mM}$ IPTG, $0.7 \%$ arabinose) is added to agarose powder $(1 \mathrm{~g})$. To dissolve the agarose, the mixture is heated to $65^{\circ} \mathrm{C}$ and frequently mixed by pipetting. The solution is then pipetted (quickly since dropping too far below $65^{\circ} \mathrm{C}$ will cause the gel to solidify in the pipette) into a perfusion chamber (already affixed onto a cover glass with Loctite). After a few minutes, the plastic sticker covering the chamber is removed and $5-7 \mu \mathrm{L}$ of a cell culture solution (OD600 of $\approx 0.003$ ) is immediately applied to the top of the exposed agarose gel and then sandwiched using (another) cover glass (see Fig. 5-4). The perfusion chamber is placed upon the heater stand, which is controlled by the heater controller to ensure that the cells are at $37^{\circ} \mathrm{C}$.

Ideally, the cells would be trapped between the cover glass and the agarose gel. However, it was found that due to the lack of gel rigidity, there was often just enough space in the vertical direction for most cells to traverse, making it difficult to maintain focus. Occasionally, as before, we would get lucky and would be able to maintain focus on a particular cell and its daughter cells, but since this was unreliable and certainly not reproducible, this protocol was abandoned in favor of using microfluidics.

\subsubsection{Microfluidics protocol}

CellASIC ONIX microfluidic plates were found to effectively trap cells. The complementary platform to run the plates offered three primary features of interest: 


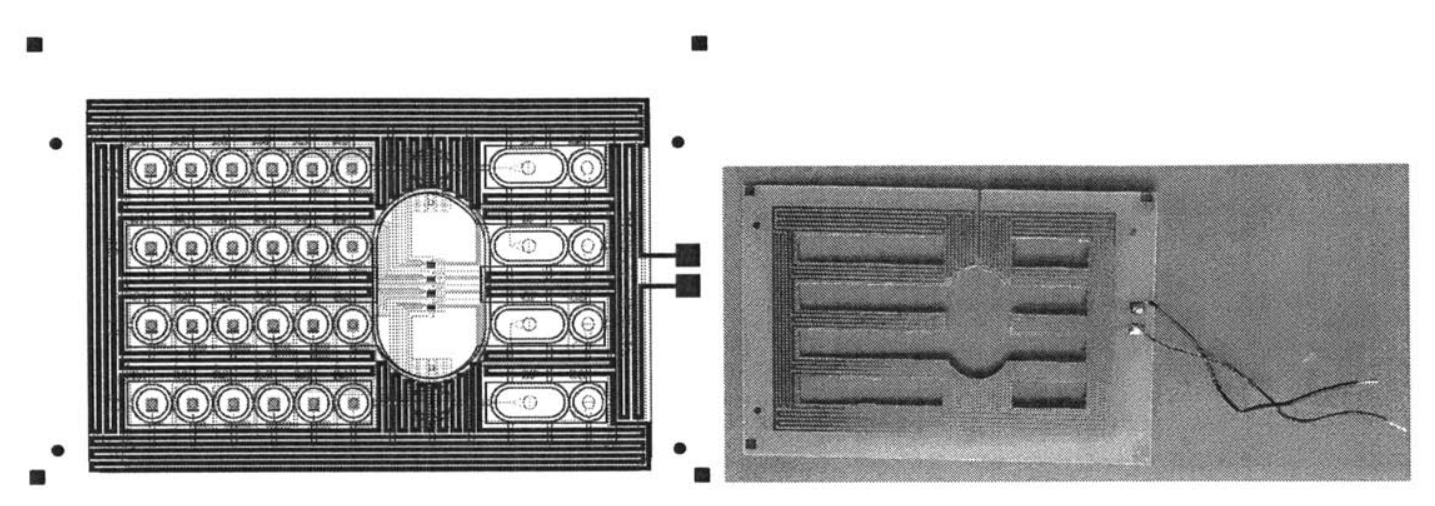

Figure 5-5: Left: PCB design (credit: Dr. Hsin-Ho Huang) overlaid with CellASIC ONIX microfluidic plate schematic. Right: Black wires correspond to the copper wires in the actual board (fabricated by Dr. Hsin-Ho Huang and Nithin Kumar).

temperature control, loading of cells and media, and flow rate regulation. Precise temperature control was achieved by air convection (using a heat exchanger). An additional advantage of heating by convention (rather than conduction) is to provide an unobstructed view of the cells in the chamber viewing window. The microfluidic plate and manifold are sealed during experimentation and loading of cells and media flow rate is pneumatically regulated (i.e., air pressure driven flow). Unfortunately, we did not have access to the CellASIC ONIX microfluidic platform and so we attempted to replicate the three aforementioned features by our own methods.

It was imperative to ensure that the cells and media were at $37^{\circ} \mathrm{C}$ throughout experimentation. To facilitate this, we designed and built a PCB (see Appendix E for protocol) and interfaced it with a temperature controller. The initial plan was to affix 2 PCBs, one over and one under the microfluidic plate and use screws to firmly hold all 3 in place. It was found that the bottom PCB (which would be in contact with the microscope stage during operation) induced small vibrations as it fluctuated in temperature around the set point temperature. We hypothesized that the metal stage acts like a heat sink, causing the temperature of the $\mathrm{PCB}$ and plate to drop too quickly once the temperature controller (which uses bang-bang control) switches the heater off. Subsequently, once switched on, the PCB takes long to reach the set point temperature. This cyclical heating and cooling cause micrometer scale vibrations that shift the focal plane of the microscope objective. Therefore, we decided to only use 

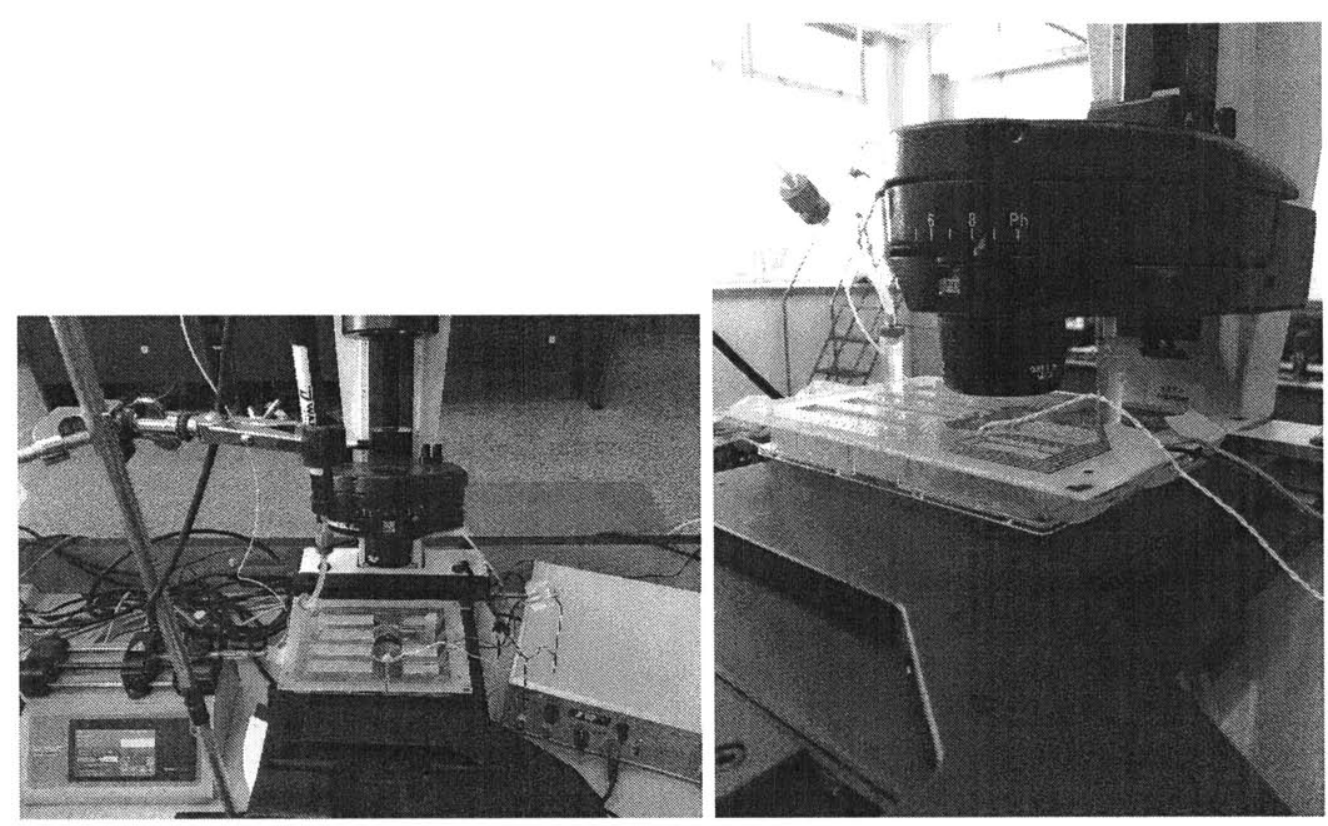

Figure 5-6: Left: Experimental setup including pump (purple, left), heater controller (white, right) with $\mathrm{PCB}$ heater affixed onto the microfluidic plate (center), which rests on the microscope stage. Right: Close-up of PCB heater and microfluidic plate with sealed cell culture suspension (clear, plastic tube on the right) and thermocouple (white wire) for heater controller.

the top PCB, which minimized temperature fluctuations and significantly improved time-lapse imagery. A schematic of the top PCB overlaid with the microfluidic plate is given in Fig. 5-5. Since we needed access to the wells (to load cells and media) and the chamber viewing window needed to be unobstructed, holes were cut using a bow saw.

The PCB is intended to act as a spatially uniform heat source and so the thick, black lines (copper wires in the actual PCB) were designed to cover as much surface area as possible. The two black squares connected to the ends of the black lines on the right are the positive and ground terminals. The final setup (Fig. 5-6) includes the pump, which controls the media and inducer solution flow rate. The tube from the syringe runs through the inline solution heater (black, vertically aligned cylinder) and feeds to an inlet port on the microfluidic plate. The port on the right of the plate is filled with cell culture suspension and is sealed after loading is complete.

During experimentation, the entire microscope (along with the microfluidic plate) 


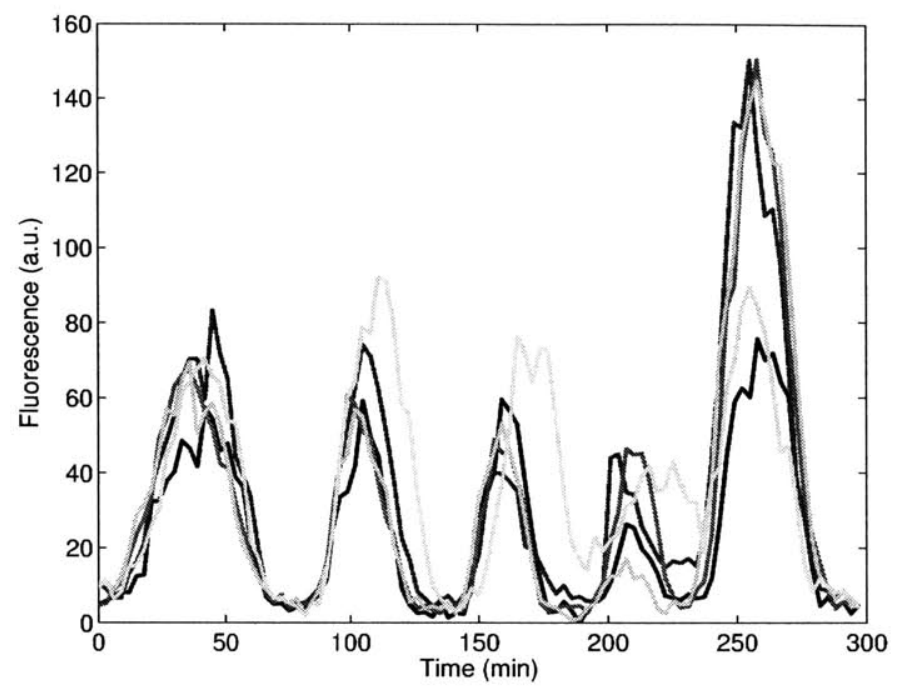

Figure 5-7: Single-cell fluorescence trajectories for 5 randomly selected cells for 5 hours.

was enclosed in a black cover to ensure no background light could affect the quality of the images. The microscope (Leica DMI6000 B inverted fluorescence microscope) was controlled by LAS AF software and imaging was performed using oil immersed $40 \mathrm{x}$ objective.

\subsection{Fluorescence data}

Single-cell fluorescence trajectories were calculated using ImageJ. The media flow rate was set to $1 \mu \mathrm{L} / \mathrm{hr}$ for relatively slow dividing time $(45 \mathrm{~min}$ ). Cells were induced with $0.7 \%$ arabinose and $2 \mathrm{mM}$ IPTG. Time-lapse images (bright-field and fluorescence) in $30 \mathrm{~min}$ intervals for a fixed region are given in Fig. 5-8. Five randomly selected cells from the same experiment were tracked for five hours and their fluorescence trajectories are plotted in Fig. 5-7. Raw data and calculations of the fluorescence is given in Appendix F.

For this particular media flow rate and inducer concentration, the oscillation period was found to be roughly 1 hour and the trajectories are in phase for the length of the experiment. The amplitude is the average gray scale intensity $(0=$ black, 255 

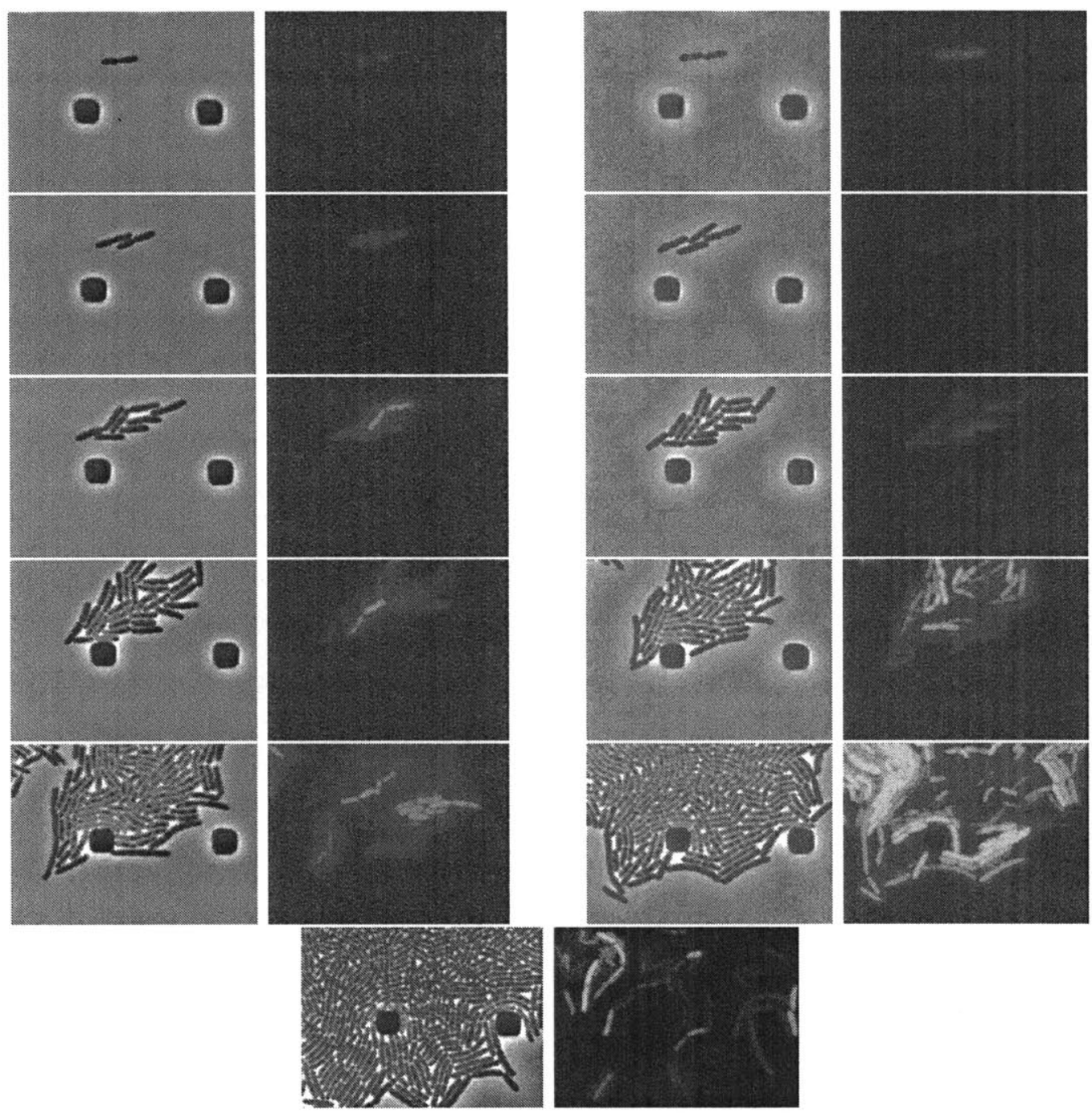

Figure 5-8: Time-lapse bright-field and green fluorescence images of oscillator using the microfluidic protocol. Images are at 30 min intervals (i.e., top left pair of images are bright-field, left, and fluorescence, right, at $\mathrm{t}=0 \mathrm{~min}$ ) for a total time of $5 \mathrm{hrs}$. 
$=$ white) of a cell calculated by finding the total fluorescence intensity of a cell and dividing by the number of pixels in the cell. The first three waves of oscillation are fairly consistent in period and amplitude across the five cells, but the amplitude of the fourth and fifth oscillations are roughly half and twice of the original amplitude oscillations, respectively. This strong amplitude oscillation late in experimentation is consistent with the results in [9] for the same inducer concentrations, confirming that our experimental setup is functional. 


\section{Chapter 6}

\section{Conclusion and Future Work}

A deterministic ODE model of an activator-repressor clock was derived from biochemical reactions to determine conditions for a stable limit cycle. The effects of load on the oscillator indicate that robust, sustained oscillations are achieved when there is strong activation and comparatively weak repression. Loading provides a means to tune the relative strengths of the activation and repression branches by changing the number of downstream DNA binding sites for either the activator or repressor protein, respectively. We have shown it is possible to activate a quenched oscillator by sequestering enough repressor protein, effectively slowing repression dynamics. Similar conclusions were reached in [21], for an activator-repressor clock with no selfrepression dynamics: in fact, the effect of retroactivity was found to be qualitatively similar with respect to the change in the expression of the trace of the linearized reduced model. This suggests that the qualitative behavior of genetic networks where there is interplay of positive and negative feedback may be effectively tuned by appropriately adjusting loads to the network's transcription factors.

The oscillator motif studied here is more robust to the effects of downstream load than the clock in [21]. For similar parameter values, the model in [23] predicts activation of a quenched oscillator with $40 \mathrm{nM}$ downstream load, but Fig. 4-2 suggests that similar activation requires $800 \mathrm{nM}$ downstream load. This is due to the additional self-repression of the repressor protein, which appears as the third term in the trace of the linearized system given in (2.15) and is always negative. Furthermore, complex 
decay of transcription factors bound to downstream binding sites degrades the performance of the oscillator. A quenched clock with decay does not exhibit oscillations when loading the repression branch and significantly less load quenches a functioning oscillator with decay when loading the activator protein.

A cost-effective, functional protocol was developed and tested for imaging the oscillator in a strain of E. coli. Fluorescence trajectories of the unloaded clock complement the results in [9]. Currently, a "load plasmid", which contains binding sites for the activator protein is being cloned. Three forms of the load plasmid (negative control, medium, and high copy number) will be transformed in the strain with the oscillator and then tested using the same microfluidics setup to observe if oscillations can be quenched for sufficiently high enough load plasmid copy number. This will be repeated for a repressor protein load plasmid to see if the fluorescence oscillation amplitude can be enhanced when sufficient repressor protein is sequestered.

The ability to tune the strength of the clock for stronger or weaker oscillations using additional DNA binding sites is useful in the context of synthetic circuits since it is easier to implement than changing the promoter regions or using degradation tags. Furthermore, this mechanism may already be used in natural systems: transcription factors have multiple DNA binding sites, not all of which serve regulatory functions [31]. One possible use of these binding sites could be to tune the dynamics of transcription networks. 


\section{Appendix A}

\section{Four-State Model Derivation of Activator-Repressor Genetic Oscillator}

A deterministic ODE model of an activator-repressor (A-R) genetic oscillator is derived considering the biochemical reactions of activation, repression, multimerization, transcription, and translation of a generic protein $(\mathrm{P})$ which, due to the symmetry of the model (both proteins are activated by $A$ and repressed by $R$ ) can be used to describe the dynamics of the concentration of both $\mathrm{A}$ and $\mathrm{R}$. These reactions are 
given by:

$$
\begin{aligned}
\mathrm{A}+\mathrm{A}+\ldots+\mathrm{A} \underset{\beta_{\mathrm{A}^{\prime}}}{\stackrel{\beta_{\mathrm{A}}}{\rightleftharpoons}} \mathrm{A}_{n}, \\
\mathrm{R}+\mathrm{R}+\ldots+\mathrm{R} \underset{\beta_{\mathrm{R}^{\prime}}}{\stackrel{\beta_{\mathrm{R}}}{\rightleftharpoons}} \mathrm{R}_{m}, \\
\mathrm{R}_{m}+\mathrm{DNA}^{\mathrm{P}} \stackrel{\mathrm{a}^{*}}{\underset{\mathrm{d}^{*}}{\rightleftharpoons}} \mathrm{R}_{m}: \mathrm{DNA}^{\mathrm{P}}, \\
\mathrm{A}_{n}+\mathrm{DNA}^{\mathrm{P}} \stackrel{\stackrel{\mathrm{a}^{\prime}}{\rightleftharpoons}}{\stackrel{\mathrm{d}^{\prime}}{\rightleftharpoons}} \mathrm{A}_{n}: \mathrm{DNA}^{\mathrm{P}}, \\
\mathrm{A}_{n}: \mathrm{DNA}^{\mathrm{P}} \stackrel{\alpha_{1}}{\longrightarrow} \mathrm{m}_{\mathrm{P}}+\mathrm{A}_{n}: \mathrm{DNA}^{\mathrm{P}}, \\
\mathrm{DNA}^{\mathrm{P}} \stackrel{\alpha_{2}}{\longrightarrow} \mathrm{m}_{\mathrm{P}}+\mathrm{DNA}^{\mathrm{P}}, \\
\mathrm{m}_{\mathrm{P}} \stackrel{\kappa}{\longrightarrow} \mathrm{m}_{\mathrm{P}}+\mathrm{P}, \\
\mathrm{m}_{\mathrm{P}} \stackrel{\delta}{\longrightarrow} \emptyset, \\
\mathrm{P} \stackrel{\gamma}{\longrightarrow} \emptyset .
\end{aligned}
$$

Let $\mathrm{A}$ and $\mathrm{R}$ multimerize with cooperativity $\mathrm{n}$ and $\mathrm{m}$, with forward rates of $\beta_{A}, \beta_{R}$ and reverse rates of $\beta_{A}^{\prime}, \beta_{R}^{\prime}$, respectively, leading to reactions (A.1)-(A.2). Since activation and repression are assumed to take place at the transcriptional level, the complex formed by the reversible reaction (with forward rate $a^{*}$ and reverse rate $d^{*}$ ) between $R_{m}$ and $D N A$ promoter $\left(D^{2} A^{P}\right.$ ), denoted $R_{m}: D N A{ }^{P}$, does not contribute to transcription and effectively sequesters free $\mathrm{DNA}^{\mathrm{P}}$, as given in (A.3). Conversely, $\mathrm{A}_{\mathrm{n}}: \mathrm{DNA}^{\mathrm{P}}$ is the complex formed by the reversible reaction (with forward rate $a^{\prime}$ and reverse rate $d^{\prime}$ ) between $\mathrm{A}_{\mathbf{n}}$ and $\mathrm{DNA}^{\mathrm{P}}$, as shown in (A.4). This complex undergoes translation at rate $\alpha_{1}$ to produce an mRNA molecule, leading to (A.5). The model also assumes that some transcription can occur without $A_{n}$ bound to DNA $^{P}$ (i.e., transcriptional leakiness), described by (A.6). Translation occurs at a rate $\kappa$, given in (A.7), and mRNA and protein decay at a rate $\delta$ and $\gamma$, respectively, given in (A.8)-(A.9). The ODE model for the mRNA and protein dynamics is given by:

$$
\begin{aligned}
\dot{m}_{P} & =\alpha_{1}\left[A_{n}: D N A^{P}\right]+\alpha_{2}\left[D N A^{P}\right]-\delta m_{P} \\
\dot{P} & =\kappa m_{P}-\gamma P .
\end{aligned}
$$


Assuming the total concentration of DNA is constant, the following conservation law holds:

$$
D N A_{t o t}=D N A^{P}+\left[R_{m}: D N A^{P}\right]+\left[A_{n}: D N A^{P}\right]
$$

Assuming complex formation occurs significantly faster than mRNA and protein dynamics [24], setting their respective rate equations at quasi-steady state (i.e., $\dot{A}_{n}, \dot{R}_{m}$, $\left.\left[\mathrm{A}_{\mathrm{n}}: \dot{\mathrm{DNA}} \mathrm{P}^{\mathrm{P}}\right],\left[\mathrm{R}_{\mathrm{m}}: \dot{\mathrm{DNA}}^{\mathrm{P}}\right]=0\right)$ and solving for $\left[\mathrm{A}_{\mathrm{n}}: \mathrm{DNA}^{\mathrm{P}}\right]$ and $\left[\mathrm{DNA}^{\mathrm{P}}\right]$ in terms of $A, R$ yields:

$$
\begin{aligned}
{\left[\mathrm{A}_{n}: \mathrm{DNA}^{\mathrm{P}}\right] } & =\frac{\frac{a^{\prime} \beta_{A}}{d^{\prime} \beta_{A^{\prime}}} D N A_{t o t} A^{n}}{1+\frac{a^{\prime} \beta_{A}}{d^{\prime} \beta_{A^{\prime}}} A^{n}+\frac{a^{*} \beta_{R}}{d^{*} \beta_{R^{\prime}}} R^{m}}, \\
{\left[\mathrm{DNA}^{\mathrm{P}}\right] } & =\frac{D N A_{t o t}}{1+\frac{a^{\prime} \beta_{A}}{d^{\prime} \beta_{A^{\prime}}} A^{n}+\frac{a^{*} \beta_{R}}{d^{*} \beta_{R^{\prime}}} R^{m}}
\end{aligned}
$$

Equation (A.10) represents the dynamics of a general mRNA and protein system with transcriptional activation and repression by $A$ and $R$, respectively. Substituting (A.11)-(A.12) in (A.10) and then using the subscripts "R" or "A" to denote parameters corresponding to $\mathrm{R}$ or A production and decay, respectively yields the final model equations:

$$
\begin{aligned}
\dot{m}_{A} & =\frac{\alpha\left(A / k_{A}\right)^{n}+\alpha_{0}}{1+\left(A / k_{A}\right)^{n}+\left(R / k_{R}\right)^{m}}-\delta_{A} m_{A} \\
\dot{m}_{R} & =\frac{\alpha\left(A / k_{A}\right)^{n}+\alpha_{0}}{1+\left(A / k_{A}\right)^{n}+\left(R / k_{R}\right)^{m}}-\delta_{R} m_{R} \\
\dot{A} & =\kappa_{A} m_{A}-\gamma_{A} A \\
\dot{R} & =\kappa_{R} m_{R}-\gamma_{R} R .
\end{aligned}
$$

In terms of the parameters used in the biochemical reactions, $\alpha=\alpha_{1} D N A_{t o t}, \alpha_{0}=$ $\alpha_{2} D N A_{t o t}, k_{A}=\left(\frac{d^{\prime} \beta_{A^{\prime}}}{a^{\prime} \beta_{A}}\right)^{\frac{1}{n}}$, and $k_{R}=\left(\frac{d^{*} \beta_{R^{\prime}}}{a^{*} \beta_{R}}\right)^{\frac{1}{m}}$. 


\section{Appendix B}

\section{Model Reduction via Singular}

\section{Perturbation for Two-State System with downstream load}

We consider A transcriptionally regulating downstream promoter sites. Let the free promoter sites be denoted as $C_{10}$ and the sites bound to $\mathrm{A}$ be denoted as $C_{11}$. Since DNA does not decay, the total concentration of promoter sites is conserved, that is $C_{10}+C_{11}=C_{t 1}$, where $C_{t 1}$ represents the total concentration of the free and bound promoter sites. The complex formation reaction is given by: $C_{10}+\underset{d}{\stackrel{a}{\rightleftarrows}} C_{11}$, leading to the three-state system:

$$
\begin{aligned}
\dot{A} & =\frac{\kappa_{A}}{\delta_{A}} \frac{\alpha\left(A / k_{A}\right)^{n}+\alpha_{0}}{1+\left(A / k_{A}\right)^{n}+\left(R / k_{R}\right)^{m}}-\gamma_{A} A-\dot{C}_{11}, \\
\dot{R} & =\frac{\kappa_{R}}{\delta_{R}} \frac{\alpha\left(A / k_{A}\right)^{n}+\alpha_{0}}{1+\left(A / k_{A}\right)^{n}+\left(R / k_{R}\right)^{m}}-\gamma_{R} R, \\
\dot{C}_{11} & =a\left(C_{t 1}-C_{11}\right) A-d C_{11} .
\end{aligned}
$$

In order to analyze how the eigenvalues of the linearized system change due to the addition of $C_{t 1}$, we consider a reduced order model. Using the assumption that complex formation $\left(\mathrm{C}_{11}\right)$ occurs relatively faster than protein dynamics $(A, R)[24]$, the three-state system can be reduced to two states. To this end, we employ singular 
perturbation and introduce the new (slow) variable $Z$, defined as $Z=A+C_{11}$. Rewrite the system by defining $\epsilon=\frac{\gamma_{A}}{d}, K_{d 1}=\frac{d}{a}$, and $a=\frac{\gamma_{A}}{\epsilon K_{d 1}}$. Substituting these expressions into (B.1) yields the system in standard singular perturbation form given by:

$$
\begin{aligned}
\dot{Z} & =\frac{\kappa_{A}}{\delta_{A}} \frac{\alpha\left(\frac{Z-C_{11}}{k_{A}}\right)^{n}+\alpha_{0}}{1+\left(\frac{Z-C_{11}}{k_{A}}\right)^{n}+\left(R / k_{R}\right)^{m}}-\gamma_{A}\left(Z-C_{11}\right), \\
\dot{R} & =\frac{\kappa_{R}}{\delta_{R}} \frac{\alpha\left(\frac{Z-C_{11}}{k_{A}}\right)^{n}+\alpha_{0}}{1+\left(\frac{Z-C_{11}}{k_{A}}\right)^{n}+\left(R / k_{R}\right)^{m}}-\gamma_{R} R, \\
\epsilon \dot{C}_{11} & =\frac{\gamma_{A}}{K_{d 1}}\left(C_{t 1}-C_{11}\right)\left(Z-C_{11}\right)-\gamma_{A} C_{11} .
\end{aligned}
$$

Setting $\epsilon=0$ and solving for $\mathrm{C}_{11}$ in terms of $\mathrm{A}$ yields the slow manifold:

$$
C_{11}=\frac{C_{t 1} A / K_{d 1}}{1+A / K_{d 1}}=g_{1}(A)
$$

which can be shown to be locally exponentially stable [30]. Since $Z=A+C_{11}$, we have $\dot{Z}=\dot{A}+\dot{C}_{11}$, and so:

$$
\dot{Z}=\dot{A}+\frac{d g_{1}(A)}{d A} \dot{A}
$$

Solving for $\dot{A}$ yields:

$$
\begin{aligned}
\dot{A} & =\frac{\dot{Z}}{1+\frac{d g_{1}(A)}{d A}}, \\
& =\left(\frac{\kappa_{A}}{\delta_{A}} \frac{\alpha\left(\frac{A}{k_{A}}\right)^{n}+\alpha_{0}}{1+\left(\frac{A}{k_{A}}\right)^{n}+\left(\frac{R}{k_{R}}\right)^{m}}-\gamma_{A} A\right) \frac{\left(1+\frac{A}{K_{d 1}}\right)^{2}}{\left(1+\frac{A}{K_{d 1}}\right)^{2}+\frac{C_{t 1}}{K_{d 1}}} .
\end{aligned}
$$

The resulting reduced model of the clock with load on A is thus given by:

$$
\begin{aligned}
& \dot{A}=\frac{\left(1+\frac{A}{K_{d 1}}\right)^{2}}{\left(1+\frac{A}{K_{d 1}}\right)^{2}+\frac{C_{t 1}}{K_{d 1}}}\left(\frac{\kappa_{A}}{\delta_{A}} \frac{\alpha\left(\frac{A}{k_{A}}\right)^{n}+\alpha_{0}}{1+\left(\frac{A}{k_{A}}\right)^{n}+\left(\frac{R}{k_{R}}\right)^{m}}-\gamma_{A} A\right), \\
& \dot{R}=\frac{\kappa_{R}}{\delta_{R}} \frac{\alpha\left(A / k_{A}\right)^{n}+\alpha_{0}}{1+\left(A / k_{A}\right)^{n}+\left(R / k_{R}\right)^{m}}-\gamma_{R} R .
\end{aligned}
$$

Model reduction of the system with load on $\mathrm{R}$ can be similarly derived. 


\section{Appendix C}

\section{Model Reduction via Singular}

\section{Perturbation for Two-State System \\ with downstream load and complex}

\section{decay}

We consider load to A transcriptionally regulating downstream promoter sites that decay at a constant rate when bound with $\mathrm{A}$. The modified load reactions to A are given by:

$$
\begin{aligned}
& A+C_{10} \underset{d}{\stackrel{a}{\rightleftarrows}} C_{11}, \\
& C_{11} \stackrel{\pi_{A}}{\rightarrow} C_{10} .
\end{aligned}
$$

The dynamics of the three-state system have changed to $\left(f_{1}(A, R)=\frac{\kappa_{A}}{\delta_{A}} \frac{\alpha\left(A / k_{A}\right)^{n}+\alpha_{0}}{1+\left(A / k_{A}\right)^{n}+\left(R / k_{R}\right)^{m}}\right.$, $\left.f_{2}(A, R)=\frac{\kappa_{R}}{\delta_{R}} \frac{\alpha\left(A / k_{A}\right)^{n}+\alpha_{0}}{1+\left(A / k_{A}\right)^{n}+\left(R / k_{R}\right)^{m}}\right):$

$$
\begin{aligned}
\dot{A} & =f_{1}(A, R)-\gamma_{A} A+d C_{11}-a A\left(C_{t 1}-C_{11}\right), \\
\dot{R} & =f_{2}(A, R)-\gamma_{R} R \\
\dot{C}_{11} & =a A\left(C_{t 1}-C_{11}\right)-\left(d+\pi_{A}\right) C_{11} .
\end{aligned}
$$


Introduce a slow $(\mathrm{X})$ and fast $(\mathrm{Y})$ variable, given by:

$$
\begin{aligned}
& X=A+C_{11}, \\
& Y=\frac{d}{d+\pi_{A}} C_{11} .
\end{aligned}
$$

The three-state system is thus now given by $\left(p_{A}=\frac{d}{d+\pi_{A}}\right)$ :

$$
\begin{aligned}
\dot{X} & =f_{1}\left(X-\frac{Y}{p_{A}}, R\right)-\gamma_{A}\left(X-\frac{Y}{p_{A}}\right)-\frac{\pi_{A} Y}{p_{A}} \\
\dot{Y} & =p_{A} a\left(X-\frac{Y}{p_{A}}\right)\left(C_{t 1}-\frac{Y}{p_{A}}\right)-d \frac{Y}{p_{A}} \\
\dot{R} & =f_{2}\left(X-\frac{Y}{p_{A}}, R\right)-\gamma_{R} R .
\end{aligned}
$$

Define $\epsilon=\frac{\gamma_{A}}{d}, K_{d 1}=\frac{d}{a}$. This leads to $d=\frac{\gamma_{A}}{\epsilon}, a=\frac{\gamma_{A}}{\epsilon K_{d 1}}$, and:

$$
\epsilon \dot{Y}=\frac{\gamma_{A} p_{A}}{K_{d 1}} A\left(C_{t 1}-\frac{Y}{p_{A}}\right)-\frac{\gamma_{A} Y}{K_{d 1}}
$$

Set $\epsilon=0$ to find the slow manifold:

$$
\begin{aligned}
Y & =\frac{p_{A}^{2}}{K_{d 1}} A\left(C_{t 1}-\frac{Y}{p_{A}}\right) \\
& =\frac{p_{A}^{2} C_{t 1} A}{K_{d 1}+p_{A} A}=h_{1}(A)
\end{aligned}
$$

Solving for $\dot{A}$ :

$$
\begin{aligned}
& X=A+\frac{Y}{p_{A}} \\
& \dot{X}=\dot{A}+\frac{1}{p_{A}} \frac{\partial h_{1}}{\partial A} \dot{A} \Longrightarrow \dot{A}=\frac{\dot{X}}{1+\frac{1}{p_{A}} \frac{\partial h_{1}}{\partial A}} \\
& \dot{A}=\frac{f_{1}(A, R)-\gamma_{A} A-\frac{\pi_{A} p_{A} C_{t 1} A}{K_{d 1}+p_{A} A}}{1+\frac{p_{A} K_{d 1} C_{t 1}}{\left(K_{d 1}+p_{A} A\right)^{2}}}
\end{aligned}
$$

Model reduction of the system with load on $\mathrm{R}$ and complex decay can be similarly derived. 


\section{Appendix D}

\section{Cell and plasmid transformation}

\section{protocol}

A sample of JS006 (gifted to us by the Biodynamics Laboratory at the University of California, San Diego) was incubated in $\mathrm{LB}$ with shaking $\left(37^{\circ} \mathrm{C}, 250 \mathrm{rpm}\right)$ until the OD600 was roughly (but not exceeding) 0.3 . An appropriate volume of the cell culture suspension was then diluted in a solution of $50 \mathrm{~mL} \mathrm{LB}, 50 \mu \mathrm{L}$ Ampicillin (Amp), and $50 \mu \mathrm{L}$ Kanamycin $(\mathrm{Km})$ for a final OD600 of 0.01 . The $50 \mathrm{~mL}$ cell culture suspension is centrifuged for $10 \mathrm{~min}\left(4^{\circ} \mathrm{C}, 3000 \mathrm{rpm}\right)$. The supernatant is discarded and pipetting is used to ensure all excess liquid is removed. An ice container was prepared and a solution of CCMB80 buffer was allowed to equilibrate to $0^{\circ} \mathrm{C}$. The precipitate (cells) was placed in the same ice container and was resuspended in $16 \mathrm{~mL}$ of ice cold CCMB80 buffer. This step required patience since hasty resuspension would potentially reduce the yield of competent cells. After resuspension was complete, the solution (cells and buffer) was centrifuged for $10 \mathrm{~min}\left(4^{\circ} \mathrm{C}, 3000 \mathrm{rpm}\right)$. Supernatant was discarded and the cells were resuspended in $1 \mathrm{~mL}$ of CCMB80 buffer to yield the competent cells. Finally, for storage, the solution was aliquoted and stored in $-80^{\circ} \mathrm{C}$. For plasmid transformation, $50 \mu \mathrm{L}$ of competent cells were removed from $-80^{\circ} \mathrm{C}$ storage and thawed on ice for 15-30 min. $1 \mu \mathrm{L}$ of pJS167 and pJS169 (purchased from Addgene, catalog \#48881 and \#48882, respectively) each was added to the competent cell solution. The solution was incubated on ice for $30 \mathrm{~min}$ and then subjected 
to heat shock treatment for $1 \mathrm{~min}\left(42^{\circ} \mathrm{C}\right) .900 \mu \mathrm{L} \mathrm{LB}$ media was added and the transformed cells were incubated with shaking at $37^{\circ} \mathrm{C}(1$ hour, $250 \mathrm{rpm})$. Plating beads were placed on a LB agar plate $(1 \mu \mathrm{L} / \mathrm{mL}$ Amp, $\mathrm{Km})$ and $50 \mu \mathrm{L}$ of the cell culture suspension was added to the middle of the plate. After shaking (to ensure cells have roughly dispersed throughout the surface of the LB plate), the beads were discarded. The plate was then sealed using Parafilm and incubated overnight $\left(37^{\circ} \mathrm{C}\right)$ without shaking yielding several transformed cell colonies. 


\section{Appendix E}

\section{PCB design and construction protocol}

The PCB was designed using Inkscape (given in Fig. 5-5) and printed on transparency film using a laser printer. The white protective coating on a MG Chemicals 600 Series presensitized copper clad boards $(150 \mathrm{~mm} \times 100 \mathrm{~mm})$ was removed and the board was placed (copper side up) under the center of a light source (40W incandescent light bulb and standard fluorescent room lighting) with the PCB film, positive side face down on the copper side for $25 \mathrm{~min}$. The copper and PCB film were held firmly in position by placing both of them in an emptied photo frame with clear glass (that is transparent to ultraviolet light).

The board was then placed in a plastic tray of 10 parts water, 1 part MG Chemicals No. 418 Developer solution. The resist (top green layer) was brushed off lightly with a foam brush until the PCB design appeared on the board, which roughly took $5 \mathrm{~min}$. Once development was completed, the now brownish/red board was rinsed with sterilized water. Ferric chloride (MG Chemicals No. 415), an effective etchant, was poured into a plastic container to sufficiently immerse the board. The etchant was heated to $50^{\circ} \mathrm{C}$ and agitation was induced (by using a foam brush and/or a fluid shaker) to decrease the time to remove the unwanted copper. After $20 \mathrm{~min}$, the finished board was once again rinsed with sterilized water. Two wires were then soldered on the ends for interfacing with the heater controller. 


\section{Appendix F}

\section{Fluorescence calculation}

A group of cells and their daughter cells were imaged using the LAS AF software (40x/1.6 magnification) at 3 min intervals in bright-field and green fluorescence modes. The images were exported as .tif files and the fluorescence images were analyzed using ImageJ. The freehand line tool was used to outline the desired cell in the first frame. The "Mean gray value" intensity, which represents the average fluorescence of the cell (i.e., total fluorescence in the outlined area divided by number of pixels), was measured for this cell outline. An arbitrary shape (say, a circle) was drawn next to the cell of interest and its intensity was measured. This serves as the background fluorescence for that image. This process is repeated for all subsequent fluorescence images of the same cell. The background mean fluorescence value is subtracted from the cell mean fluorescence value, yielding the corrected mean cell fluorescence. The raw data of the cell mean fluorescence (Cell), background mean fluorescence (Background), and their difference (Corrected) are given in the following tables.

\begin{tabular}{|c|c|c|c|}
\hline Time $(\mathrm{min})$ & Cell & Background & Corrected \\
\hline 0 & 29.68 & 24.87 & 4.81 \\
\hline 3 & 29.52 & 23.71 & 5.81 \\
\hline 6 & 31.41 & 24.36 & 7.05 \\
\hline 9 & 32.68 & 24.38 & 8.30 \\
\hline 12 & 31.79 & 23.78 & 8.01 \\
\hline 15 & 36.07 & 23.65 & 12.42 \\
\hline 18 & 36.27 & 23.51 & 12.76 \\
\hline 21 & 56.75 & 24.19 & 32.56 \\
\hline 24 & 55.79 & 23.43 & 32.37 \\
\hline
\end{tabular}




\begin{tabular}{|c|c|c|c|}
\hline 27 & 60.84 & 23.04 & 37.81 \\
\hline 30 & 64.31 & 24.04 & 40.28 \\
\hline 33 & 72.39 & 23.85 & 48.54 \\
\hline 36 & 70.06 & 23.62 & 46.45 \\
\hline 39 & 64.5 & 22.78 & 41.72 \\
\hline 42 & 80.76 & 23.44 & 57.32 \\
\hline 45 & 77.73 & 23.44 & 54.3 \\
\hline 48 & 69.46 & 23.52 & 45.94 \\
\hline 51 & 71.72 & 23.87 & 47.85 \\
\hline 54 & 61.64 & 22.75 & 38.88 \\
\hline 57 & 59.93 & 23.59 & 36.35 \\
\hline 60 & 44.48 & 23.01 & 21.47 \\
\hline 63 & 39.03 & 23.73 & 15.3 \\
\hline 66 & 30.92 & 24.03 & 6.89 \\
\hline 69 & 28.73 & 23.7 & 5.03 \\
\hline 72 & 28.61 & 23.43 & 5.18 \\
\hline 75 & 26.77 & 23.55 & 3.22 \\
\hline 78 & 28.31 & 24.19 & 4.12 \\
\hline 81 & 27.21 & 23.68 & 3.53 \\
\hline 84 & 28.56 & 23.21 & 5.35 \\
\hline 87 & 30.57 & 23.04 & 7.53 \\
\hline 90 & 35.63 & 24 & 11.64 \\
\hline 93 & 48.11 & 23.35 & 24.76 \\
\hline 96 & 57.02 & 23.39 & 33.63 \\
\hline 99 & 59.82 & 24.77 & 35.05 \\
\hline 102 & 66.21 & 24.14 & 42.07 \\
\hline 105 & 82.73 & 23.47 & 59.25 \\
\hline 108 & 72.72 & 23.3 & 49.42 \\
\hline 111 & 68.97 & 24.46 & 44.51 \\
\hline 114 & 52.87 & 24.41 & 28.46 \\
\hline 117 & 46.09 & 23.13 & 22.95 \\
\hline 120 & 32.41 & 23.32 & 9.1 \\
\hline 123 & 28.51 & 23.76 & 4.75 \\
\hline 126 & 26.18 & 23.64 & 2.54 \\
\hline 129 & 27.35 & 24.56 & 2.79 \\
\hline 132 & 25.7 & 24.08 & 1.62 \\
\hline 135 & 29.74 & 23.23 & 6.51 \\
\hline 138 & 24.99 & 23.63 & 1.35 \\
\hline 141 & 26.68 & 24.04 & 2.63 \\
\hline 144 & 25.32 & 22.98 & 2.33 \\
\hline 147 & 33.72 & 23.61 & 10.11 \\
\hline 150 & 52.23 & 23.86 & 28.37 \\
\hline 153 & 60.3 & 23.86 & 36.44 \\
\hline 156 & 63.73 & 23.24 & 40.49 \\
\hline 159 & 83.04 & 23.42 & 59.61 \\
\hline 162 & 79.91 & 23.29 & 56.62 \\
\hline 165 & 76.83 & 23.96 & 52.88 \\
\hline
\end{tabular}




\begin{tabular}{|c|c|c|c|}
\hline 168 & 61.82 & 24.35 & 37.47 \\
\hline 171 & 47 & 24.21 & 22.79 \\
\hline 174 & 35.88 & 24.69 & 11.19 \\
\hline 177 & 26.72 & 23.9 & 2.82 \\
\hline 180 & 27.55 & 24.48 & 3.07 \\
\hline 183 & 29.16 & 23.49 & 5.67 \\
\hline 186 & 26.98 & 23.77 & 3.21 \\
\hline 189 & 24.32 & 24.4 & -0.08 \\
\hline 192 & 28.35 & 24.49 & 3.86 \\
\hline 195 & 28.67 & 23.39 & 5.28 \\
\hline 198 & 32.20 & 25.28 & 6.92 \\
\hline 201 & 36.44 & 25.48 & 10.97 \\
\hline 204 & 44.63 & 26.16 & 18.47 \\
\hline 207 & 51.49 & 25.12 & 26.37 \\
\hline 210 & 51.52 & 26.15 & 25.38 \\
\hline 213 & 44.99 & 25.27 & 19.73 \\
\hline 216 & 42.68 & 25.4 & 17.28 \\
\hline 219 & 38.43 & 25.21 & 13.22 \\
\hline 222 & 33.4 & 26.18 & 7.22 \\
\hline 225 & 32.64 & 25.95 & 6.69 \\
\hline 228 & 31.77 & 25.66 & 6.11 \\
\hline 231 & 32.87 & 25.13 & 7.74 \\
\hline 234 & 31.32 & 24.77 & 6.55 \\
\hline 237 & 31.83 & 24.91 & 6.92 \\
\hline 240 & 37.62 & 25.68 & 11.94 \\
\hline 243 & 51.01 & 24.89 & 26.12 \\
\hline 246 & 71.55 & 26.33 & 45.22 \\
\hline 249 & 84.75 & 25.78 & 58.97 \\
\hline 252 & 88.9 & 26.24 & 62.67 \\
\hline 255 & 86.38 & 26.15 & 60.23 \\
\hline 258 & 101.39 & 25.64 & 75.75 \\
\hline 261 & 96.98 & 26.88 & 70.09 \\
\hline 264 & 98.59 & 26.64 & 71.95 \\
\hline 267 & 91.27 & 27.11 & 64.16 \\
\hline 270 & 86.93 & 26.55 & 60.38 \\
\hline 273 & 63.14 & 26.29 & 36.85 \\
\hline 276 & 54.77 & 26.89 & 27.88 \\
\hline 279 & 41.09 & 26.89 & 14.21 \\
\hline 282 & 31.58 & 27.05 & 4.53 \\
\hline 285 & 32.96 & 25.99 & 6.97 \\
\hline 288 & 30.31 & 26.15 & 4.16 \\
\hline 291 & 31.4 & 25.76 & 5.63 \\
\hline 294 & 29.33 & 26.84 & 2.49 \\
\hline 297 & 32.72 & 27.62 & 5.10 \\
\hline
\end{tabular}

Table F.1: Cell 1 data 


\begin{tabular}{|c|c|c|c|}
\hline Time (min) & Cell & Background & Corrected \\
\hline 0 & 35.31 & 24.87 & 10.45 \\
\hline 3 & 32.63 & 23.70 & 8.93 \\
\hline 6 & 30.93 & 24.36 & 6.57 \\
\hline 9 & 30.94 & 24.384 & 6.56 \\
\hline 12 & 37.00 & 23.78 & 13.22 \\
\hline 15 & 39.76 & 23.65 & 16.11 \\
\hline 18 & 44.45 & 23.51 & 20.95 \\
\hline 21 & 59.97 & 24.19 & 35.78 \\
\hline 24 & 66.28 & 23.43 & 42.85 \\
\hline 27 & 71.76 & 23.04 & 48.72 \\
\hline 30 & 79.25 & 24.04 & 55.21 \\
\hline 33 & 88.76 & 23.85 & 64.91 \\
\hline 36 & 94.01 & 23.62 & 70.39 \\
\hline 39 & 93.16 & 22.78 & 70.38 \\
\hline 42 & 83.19 & 23.43 & 59.75 \\
\hline 45 & 106.72 & 23.43 & 83.28 \\
\hline 48 & 95.43 & 23.52 & 71.91 \\
\hline 51 & 88.81 & 23.87 & 64.94 \\
\hline 54 & 67.18 & 22.75 & 44.43 \\
\hline 57 & 63.10 & 23.59 & 39.51 \\
\hline 60 & 56.66 & 23.01 & 33.65 \\
\hline 63 & 43.95 & 23.73 & 20.22 \\
\hline 66 & 32.84 & 24.03 & 8.81 \\
\hline 69 & 30.12 & 23.70 & 6.42 \\
\hline 72 & 29.90 & 23.43 & 6.47 \\
\hline 75 & 28.91 & 23.55 & 5.36 \\
\hline 78 & 29.31 & 24.19 & 5.13 \\
\hline 81 & 29.01 & 23.68 & 5.33 \\
\hline 84 & 30.30 & 23.21 & 7.09 \\
\hline 87 & 32.07 & 23.04 & 9.03 \\
\hline 90 & 35.97 & 24.00 & 11.97 \\
\hline 93 & 53.58 & 23.35 & 30.23 \\
\hline 96 & 65.00 & 23.39 & 41.61 \\
\hline 99 & 77.26 & 24.77 & 52.49 \\
\hline 102 & 86.67 & 24.14 & 62.52 \\
\hline 105 & 97.59 & 23.47 & 74.12 \\
\hline 108 & 94.42 & 23.30 & 71.13 \\
\hline 111 & 87.00 & 24.46 & 62.53 \\
\hline 114 & 75.40 & 24.41 & 50.99 \\
\hline 117 & 60.83 & 23.13 & 37.70 \\
\hline 120 & 45.82 & 23.32 & 22.5 \\
\hline 123 & 40.66 & 23.76 & 16.90 \\
\hline 126 & 31.93 & 23.64 & 8.29 \\
\hline 129 & 31.08 & 24.56 & 6.53 \\
\hline 132 & 28.4 & 24.08 & 4.32 \\
\hline 135 & 29.19 & 23.23 & 5.96 \\
\hline & & & \\
\hline
\end{tabular}




\begin{tabular}{|c|c|c|c|}
\hline 138 & 30.87 & 23.63 & 7.24 \\
\hline 141 & 28.87 & 24.04 & 4.82 \\
\hline 144 & 30.32 & 22.98 & 7.33 \\
\hline 147 & 34.90 & 23.61 & 11.28 \\
\hline 150 & 42.37 & 23.86 & 18.51 \\
\hline 153 & 58.18 & 23.86 & 34.32 \\
\hline 156 & 63.41 & 23.24 & 40.17 \\
\hline 159 & 63.15 & 23.42 & 39.73 \\
\hline 162 & 61.01 & 23.29 & 37.72 \\
\hline 165 & 59.14 & 23.96 & 35.18 \\
\hline 168 & 44.68 & 24.35 & 20.33 \\
\hline 171 & 43.97 & 24.21 & 19.73 \\
\hline 174 & 40.17 & 24.69 & 15.48 \\
\hline 177 & 35.15 & 23.90 & 11.25 \\
\hline 180 & 32.26 & 24.48 & 7.78 \\
\hline 183 & 33.497 & 23.49 & 10.01 \\
\hline 186 & 30.33 & 23.77 & 6.57 \\
\hline 189 & 31.436 & 24.4 & 7.04 \\
\hline 192 & 30.22 & 24.49 & 5.73 \\
\hline 195 & 29.529 & 23.39 & 6.14 \\
\hline 198 & 34.87 & 25.276 & 9.59 \\
\hline 201 & 69.57 & 25.48 & 44.1 \\
\hline 204 & 71.23 & 26.16 & 45.07 \\
\hline 207 & 59.97 & 25.12 & 34.85 \\
\hline 210 & 60.47 & 26.15 & 34.32 \\
\hline 213 & 50.91 & 25.27 & 25.64 \\
\hline 216 & 47.17 & 25.4 & 21.77 \\
\hline 219 & 43.01 & 25.21 & 17.8 \\
\hline 222 & 40.93 & 26.18 & 14.75 \\
\hline 225 & 42.49 & 25.95 & 16.54 \\
\hline 228 & 41.60 & 25.66 & 15.94 \\
\hline 231 & 42.30 & 25.13 & 17.17 \\
\hline 234 & 40.05 & 24.77 & 15.28 \\
\hline 237 & 47.68 & 24.91 & 22.76 \\
\hline 240 & 69.49 & 25.68 & 43.81 \\
\hline 243 & 92.89 & 24.89 & 68 \\
\hline 246 & 126.42 & 26.33 & 100.09 \\
\hline 249 & 159.51 & 25.78 & 133.73 \\
\hline 252 & 158.49 & 26.23 & 132.25 \\
\hline 255 & 176.57 & 26.15 & 150.42 \\
\hline 258 & 151.92 & 25.64 & 126.28 \\
\hline 261 & 135.55 & 26.88 & 108.67 \\
\hline 264 & 137.10 & 26.64 & 110.46 \\
\hline 267 & 122.55 & 27.11 & 95.45 \\
\hline 270 & 94.36 & 26.55 & 67.82 \\
\hline 273 & 64.31 & 26.29 & 38.01 \\
\hline 276 & 54.57 & 26.89 & 27.68 \\
\hline
\end{tabular}




\begin{tabular}{|c|c|c|c|}
\hline 279 & 42.04 & 26.89 & 15.15 \\
\hline 282 & 36.23 & 27.05 & 9.18 \\
\hline 285 & 34.49 & 25.99 & 8.5 \\
\hline 288 & 33.45 & 26.15 & 7.31 \\
\hline 291 & 33.89 & 25.76 & 8.13 \\
\hline 294 & 33.63 & 26.84 & 6.79 \\
\hline 297 & 32.41 & 27.622 & 4.79 \\
\hline
\end{tabular}

Table F.2: Cell 2 data

\begin{tabular}{|c|c|c|c|}
\hline Time $(\min )$ & Cell & Background & Corrected \\
\hline 0 & 35.31 & 24.87 & 10.45 \\
\hline 3 & 32.63 & 23.71 & 8.93 \\
\hline 6 & 30.93 & 24.36 & 6.57 \\
\hline 9 & 30.94 & 24.38 & 6.56 \\
\hline 12 & 37 & 23.78 & 13.22 \\
\hline 15 & 39.76 & 23.65 & 16.11 \\
\hline 18 & 44.46 & 23.51 & 20.95 \\
\hline 21 & 59.97 & 24.19 & 35.78 \\
\hline 24 & 66.28 & 23.43 & 42.85 \\
\hline 27 & 71.76 & 23.04 & 48.72 \\
\hline 30 & 79.25 & 24.04 & 55.21 \\
\hline 33 & 88.76 & 23.85 & 64.91 \\
\hline 36 & 94.01 & 23.62 & 70.39 \\
\hline 39 & 93.16 & 22.78 & 70.38 \\
\hline 42 & 83.19 & 23.44 & 59.75 \\
\hline 45 & 106.72 & 23.44 & 83.28 \\
\hline 48 & 95.43 & 23.52 & 71.91 \\
\hline 51 & 88.81 & 23.87 & 64.94 \\
\hline 54 & 67.18 & 22.75 & 44.43 \\
\hline 57 & 63.1 & 23.59 & 39.51 \\
\hline 60 & 56.66 & 23.01 & 33.65 \\
\hline 63 & 43.95 & 23.73 & 20.22 \\
\hline 66 & 32.84 & 24.03 & 8.81 \\
\hline 69 & 30.12 & 23.7 & 6.42 \\
\hline 72 & 29.9 & 23.43 & 6.47 \\
\hline 75 & 28.91 & 23.55 & 5.36 \\
\hline 78 & 29.31 & 24.19 & 5.13 \\
\hline 81 & 29.01 & 23.68 & 5.33 \\
\hline 84 & 30.3 & 23.21 & 7.09 \\
\hline 87 & 32.07 & 23.04 & 9.03 \\
\hline 90 & 35.97 & 24 & 11.97 \\
\hline 93 & 53.58 & 23.35 & 30.23 \\
\hline 96 & 65 & 23.39 & 41.61 \\
\hline 99 & 77.26 & 24.77 & 52.49 \\
\hline 102 & 86.67 & 24.14 & 62.52 \\
\hline 105 & 97.59 & 23.47 & 74.12 \\
\hline
\end{tabular}




\begin{tabular}{|c|c|c|c|}
\hline 108 & 94.43 & 23.3 & 71.13 \\
\hline 111 & 87 & 24.46 & 62.53 \\
\hline 114 & 75.40 & 24.41 & 50.99 \\
\hline 117 & 60.83 & 23.13 & 37.70 \\
\hline 120 & 45.82 & 23.32 & 22.5 \\
\hline 123 & 40.66 & 23.76 & 16.90 \\
\hline 126 & 31.93 & 23.64 & 8.29 \\
\hline 129 & 31.08 & 24.56 & 6.53 \\
\hline 132 & 28.4 & 24.08 & 4.32 \\
\hline 135 & 29.19 & 23.23 & 5.96 \\
\hline 138 & 30.87 & 23.63 & 7.24 \\
\hline 141 & 28.87 & 24.04 & 4.82 \\
\hline 144 & 30.32 & 22.98 & 7.33 \\
\hline 147 & 34.9 & 23.61 & 11.28 \\
\hline 150 & 42.37 & 23.86 & 18.51 \\
\hline 153 & 58.18 & 23.86 & 34.32 \\
\hline 156 & 63.41 & 23.24 & 40.17 \\
\hline 159 & 63.15 & 23.42 & 39.73 \\
\hline 162 & 61.01 & 23.29 & 37.72 \\
\hline 165 & 59.14 & 23.96 & 35.18 \\
\hline 168 & 44.68 & 24.35 & 20.33 \\
\hline 171 & 43.94 & 24.21 & 19.73 \\
\hline 174 & 40.17 & 24.69 & 15.48 \\
\hline 177 & 35.15 & 23.9 & 11.25 \\
\hline 180 & 32.26 & 24.48 & 7.78 \\
\hline 183 & 33.5 & 23.49 & 10.01 \\
\hline 186 & 30.33 & 23.77 & 6.57 \\
\hline 189 & 31.44 & 24.4 & 7.04 \\
\hline $19 \overline{2}$ & 30.22 & 24.49 & 5.73 \\
\hline 195 & 29.53 & 23.39 & 6.14 \\
\hline 198 & 34.87 & 25.28 & 9.59 \\
\hline 201 & 69.57 & 25.48 & 44.1 \\
\hline 204 & 71.23 & 26.16 & 45.07 \\
\hline 207 & 59.97 & 25.12 & 34.85 \\
\hline 210 & 60.47 & 26.15 & 34.32 \\
\hline 213 & 50.91 & 25.27 & 25.64 \\
\hline 216 & 47.17 & 25.4 & 21.77 \\
\hline 219 & 43.01 & 25.21 & 17.8 \\
\hline 222 & 40.93 & 26.18 & 14.75 \\
\hline 225 & 42.49 & 25.95 & 16.54 \\
\hline 228 & 41.6 & 25.66 & 15.94 \\
\hline 231 & 42.3 & 25.13 & 17.17 \\
\hline 234 & 40.05 & 24.77 & 15.28 \\
\hline 237 & 47.68 & 24.91 & 22.76 \\
\hline 240 & 69.49 & 25.68 & 43.81 \\
\hline 243 & 92.89 & 24.89 & 68 \\
\hline 246 & 126.42 & 26.33 & 100.09 \\
\hline
\end{tabular}




\begin{tabular}{|c|c|c|c|}
\hline 249 & 159.51 & 25.78 & 133.73 \\
\hline 252 & 158.49 & 26.24 & 132.25 \\
\hline 255 & 176.57 & 26.15 & 150.42 \\
\hline 258 & 151.92 & 25.64 & 126.28 \\
\hline 261 & 135.55 & 26.88 & 108.67 \\
\hline 264 & 137.1 & 26.64 & 110.46 \\
\hline 267 & 122.55 & 27.11 & 95.45 \\
\hline 270 & 94.37 & 26.55 & 67.82 \\
\hline 273 & 64.31 & 26.29 & 38.01 \\
\hline 276 & 54.57 & 26.89 & 27.68 \\
\hline 279 & 42.04 & 26.89 & 15.15 \\
\hline 282 & 36.23 & 27.05 & 9.18 \\
\hline 285 & 34.49 & 25.99 & 8.5 \\
\hline 288 & 33.45 & 26.15 & 7.31 \\
\hline 291 & 33.89 & 25.76 & 8.13 \\
\hline 294 & 33.64 & 26.84 & 6.79 \\
\hline 297 & 32.41 & 27.62 & 4.79 \\
\hline
\end{tabular}

Table F.3: Cell 3 data

\begin{tabular}{|c|c|c|c|}
\hline Time (min) & Cell & Background & Corrected \\
\hline 0 & 35.14 & 24.87 & 10.27 \\
\hline 3 & 34.30 & 23.71 & 10.59 \\
\hline 6 & 33.20 & 24.36 & 8.84 \\
\hline 9 & 36.49 & 24.38 & 12.11 \\
\hline 12 & 42.67 & 23.78 & 18.89 \\
\hline 15 & 52.18 & 23.65 & 28.53 \\
\hline 18 & 54.74 & 23.51 & 31.24 \\
\hline 21 & 59.88 & 24.19 & 35.69 \\
\hline 24 & 69.05 & 23.43 & 45.62 \\
\hline 27 & 80.05 & 23.04 & 57.01 \\
\hline 30 & 79.42 & 24.04 & 55.39 \\
\hline 33 & 88.29 & 23.85 & 64.44 \\
\hline 36 & 93.18 & 23.62 & 69.56 \\
\hline 39 & 73.49 & 22.78 & 50.71 \\
\hline 42 & 79.67 & 23.44 & 56.23 \\
\hline 45 & 81.95 & 23.44 & 58.52 \\
\hline 48 & 76.65 & 23.52 & 53.13 \\
\hline 51 & 65.63 & 23.87 & 41.75 \\
\hline 54 & 51.51 & 22.75 & 28.76 \\
\hline 57 & 44.03 & 23.59 & 20.44 \\
\hline 60 & 39.08 & 23.01 & 16.07 \\
\hline 63 & 35.37 & 23.73 & 11.63 \\
\hline 66 & 33.07 & 24.03 & 9.04 \\
\hline 69 & 30.33 & 23.7 & 6.63 \\
\hline 72 & 29.82 & 23.43 & 6.39 \\
\hline 75 & 28.75 & 23.55 & 5.21 \\
\hline & & & \\
\hline
\end{tabular}




\begin{tabular}{|c|c|c|c|}
\hline 78 & 30.62 & 24.19 & 6.43 \\
\hline 81 & 26.32 & 23.68 & 2.64 \\
\hline 84 & 28.49 & 23.21 & 5.27 \\
\hline 87 & 31.52 & 23.04 & 8.48 \\
\hline 90 & 39.33 & 24 & 15.33 \\
\hline 93 & 47.51 & 23.35 & 24.16 \\
\hline 96 & 65.66 & 23.39 & 42.27 \\
\hline 99 & 69.53 & 24.77 & 44.77 \\
\hline 102 & 81.01 & 24.14 & 56.87 \\
\hline 105 & 78.36 & 23.47 & 54.89 \\
\hline 108 & 67.51 & 23.3 & 44.21 \\
\hline 111 & 64.89 & 24.46 & 40.43 \\
\hline 114 & 59.47 & 24.41 & 35.07 \\
\hline 117 & 43.21 & 23.13 & 20.08 \\
\hline 120 & 36.97 & 23.32 & 13.65 \\
\hline 123 & 29.37 & 23.76 & 5.61 \\
\hline 126 & 30.42 & 23.64 & 6.78 \\
\hline 129 & 28.47 & 24.56 & 3.91 \\
\hline 132 & 28.51 & 24.08 & 4.44 \\
\hline 135 & 26.94 & 23.23 & 3.71 \\
\hline 138 & 28.94 & 23.63 & 5.31 \\
\hline 141 & 26.48 & 24.04 & 2.43 \\
\hline 144 & 30.02 & 22.98 & 7.04 \\
\hline 147 & 40.68 & 23.61 & 17.07 \\
\hline 150 & 57.77 & 23.86 & 33.91 \\
\hline 153 & 63.05 & 23.86 & 39.19 \\
\hline 156 & 69.07 & 23.24 & 45.83 \\
\hline 159 & 76.7 & 23.42 & 53.28 \\
\hline 162 & 69.2 & 23.29 & 45.91 \\
\hline 165 & 62.85 & 23.96 & 38.89 \\
\hline 168 & 51.74 & 24.35 & 27.39 \\
\hline 171 & 38.6 & 24.21 & 14.39 \\
\hline 174 & 30.89 & 24.69 & 6.2 \\
\hline 177 & 28.53 & 23.9 & 4.63 \\
\hline 180 & 28.63 & 24.48 & 4.15 \\
\hline 183 & 25.7 & 23.49 & 2.21 \\
\hline 186 & 24.57 & 23.77 & 0.81 \\
\hline 189 & 25.53 & 24.4 & 1.13 \\
\hline 192 & 28.65 & 24.49 & 4.16 \\
\hline 195 & 28.47 & 23.39 & 5.08 \\
\hline 198 & 34.88 & 25.28 & 9.6 \\
\hline 201 & 37.83 & 25.48 & 12.36 \\
\hline 204 & 38.89 & 26.16 & 12.73 \\
\hline 207 & 42.31 & 25.12 & 17.19 \\
\hline 210 & 37.79 & 26.15 & 11.64 \\
\hline 213 & 38 & 25.27 & 12.73 \\
\hline 216 & 34.72 & 25.4 & 9.32 \\
\hline & & & \\
\hline
\end{tabular}




\begin{tabular}{|c|c|c|c|}
\hline 219 & 29.08 & 25.21 & 3.87 \\
\hline 222 & 28.6 & 26.18 & 2.42 \\
\hline 225 & 31.78 & 25.95 & 5.83 \\
\hline 228 & 30.65 & 25.66 & 4.99 \\
\hline 231 & 30.35 & 25.13 & 5.22 \\
\hline 234 & 36.6 & 24.77 & 11.83 \\
\hline 237 & 50.76 & 24.91 & 25.85 \\
\hline 240 & 63.26 & 25.68 & 37.58 \\
\hline 243 & 95.77 & 24.89 & 70.88 \\
\hline 246 & 112.2 & 26.33 & 85.87 \\
\hline 249 & 124.83 & 25.78 & 99.05 \\
\hline 252 & 155.22 & 26.24 & 128.99 \\
\hline 255 & 164.63 & 26.15 & 138.48 \\
\hline 258 & 170.52 & 25.64 & 144.89 \\
\hline 261 & 161.93 & 26.88 & 135.05 \\
\hline 264 & 149.68 & 26.64 & 123.04 \\
\hline 267 & 149.12 & 27.11 & 122.01 \\
\hline 270 & 122.39 & 26.55 & 95.84 \\
\hline 273 & 94.02 & 26.29 & 67.73 \\
\hline 276 & 70.32 & 26.89 & 43.44 \\
\hline 279 & 48.8 & 26.89 & 21.92 \\
\hline 282 & 38.69 & 27.05 & 11.64 \\
\hline 285 & 35.69 & 25.99 & 9.70 \\
\hline 288 & 32.34 & 26.15 & 6.2 \\
\hline 291 & 34.29 & 25.76 & 8.53 \\
\hline 294 & 31.53 & 26.84 & 4.69 \\
\hline 297 & 31.98 & 27.62 & 4.36 \\
\hline & & & \\
\hline
\end{tabular}

Table F.4: Cell 4 data

\begin{tabular}{|c|c|c|c|}
\hline Time (min) & Cell & Background & Corrected \\
\hline 0 & 34.18 & 24.87 & 9.31 \\
\hline 3 & 35.02 & 23.71 & 11.31 \\
\hline 6 & 30.7 & 24.36 & 6.34 \\
\hline 9 & 32.65 & 24.38 & 8.27 \\
\hline 12 & 38.63 & 23.78 & 14.86 \\
\hline 15 & 39.01 & 23.65 & 15.36 \\
\hline 18 & 42.88 & 23.51 & 19.37 \\
\hline 21 & 50.45 & 24.19 & 26.26 \\
\hline 24 & 53.39 & 23.43 & 29.97 \\
\hline 27 & 67.64 & 23.04 & 44.61 \\
\hline 30 & 72.91 & 24.04 & 48.88 \\
\hline 33 & 84.81 & 23.85 & 60.96 \\
\hline 36 & 87.93 & 23.62 & 64.31 \\
\hline 39 & 90.41 & 22.78 & 67.64 \\
\hline 42 & 93.9 & 23.44 & 70.46 \\
\hline 45 & 88.99 & 23.44 & 65.55 \\
\hline
\end{tabular}




\begin{tabular}{|c|c|c|c|}
\hline 48 & 87.58 & 23.52 & 64.06 \\
\hline 51 & 77.63 & 23.87 & 53.76 \\
\hline 54 & 76.24 & 22.75 & 53.48 \\
\hline 57 & 64.46 & 23.59 & 40.87 \\
\hline 60 & 44.56 & 23.01 & 21.55 \\
\hline 63 & 41.11 & 23.73 & 17.37 \\
\hline 66 & 33.87 & 24.03 & 9.84 \\
\hline 69 & 30.17 & 23.7 & 6.47 \\
\hline 72 & 30.25 & 23.43 & 6.83 \\
\hline 75 & 29.01 & 23.55 & 5.46 \\
\hline 78 & 28.59 & 24.19 & 4.41 \\
\hline 81 & 25.74 & 23.68 & 2.06 \\
\hline 84 & 28.08 & 23.21 & 4.87 \\
\hline 87 & 27.74 & 23.04 & 4.71 \\
\hline 90 & 37.58 & 24 & 13.58 \\
\hline 93 & 49.96 & 23.35 & 26.6 \\
\hline 96 & 67.59 & 23.39 & 44.2 \\
\hline 99 & 85.09 & 24.77 & 60.33 \\
\hline 102 & 88.28 & 24.14 & 64.13 \\
\hline 105 & 102.2 & 23.47 & 78.73 \\
\hline 108 & 100.43 & 23.3 & 77.14 \\
\hline 111 & 116.35 & 24.46 & 91.88 \\
\hline 114 & 115.56 & 24.41 & 91.15 \\
\hline 117 & 103.13 & 23.13 & 80 \\
\hline 120 & 81.65 & 23.32 & 58.33 \\
\hline 123 & 79.16 & 23.76 & 55.41 \\
\hline 126 & 59.1 & 23.64 & 35.47 \\
\hline 129 & 45.79 & 24.56 & 21.23 \\
\hline 132 & 34.26 & 24.08 & 10.18 \\
\hline 135 & 29.7 & 23.23 & 6.47 \\
\hline 138 & 27.45 & 23.63 & 3.82 \\
\hline 141 & 27.53 & 24.04 & 3.49 \\
\hline 144 & 27.29 & 22.98 & 4.31 \\
\hline 147 & 32.79 & 23.61 & 9.18 \\
\hline 150 & 31.13 & 23.86 & 7.27 \\
\hline 153 & 38.66 & 23.86 & 14.8 \\
\hline 156 & 49.28 & 23.24 & 26.04 \\
\hline 159 & 60.79 & 23.42 & 37.37 \\
\hline 162 & 74.49 & 23.29 & 51.2 \\
\hline 165 & 100.26 & 23.96 & 76.3 \\
\hline 168 & 96.6 & 24.35 & 72.25 \\
\hline 171 & 88.36 & 24.21 & 64.15 \\
\hline 174 & 97.65 & 24.69 & 72.96 \\
\hline 177 & 96.76 & 23.9 & 72.85 \\
\hline 180 & 79.72 & 24.48 & 55.25 \\
\hline 183 & 59.4 & 23.49 & 35.9 \\
\hline 186 & 45.78 & 23.77 & 22.01 \\
\hline & & & \\
\hline
\end{tabular}




\begin{tabular}{|c|c|c|c|}
\hline 189 & 41.18 & 24.4 & 16.78 \\
\hline 192 & 38.53 & 24.49 & 14.04 \\
\hline 195 & 46.48 & 23.39 & 23.09 \\
\hline 198 & 44.57 & 25.28 & 19.3 \\
\hline 201 & 47.69 & 25.48 & 22.21 \\
\hline 204 & 49.95 & 26.16 & 23.79 \\
\hline 207 & 56.25 & 25.12 & 31.13 \\
\hline 210 & 58.62 & 26.15 & 32.47 \\
\hline 213 & 64.46 & 25.27 & 39.19 \\
\hline 216 & 67.27 & 25.4 & 41.87 \\
\hline 219 & 60.4 & 25.21 & 35.19 \\
\hline 222 & 62.29 & 26.18 & 36.11 \\
\hline 225 & 69.07 & 25.95 & 43.12 \\
\hline 228 & 63.09 & 25.66 & 37.42 \\
\hline 231 & 57.56 & 25.13 & 32.43 \\
\hline 234 & 56.79 & 24.77 & 32.02 \\
\hline 237 & 43.95 & 24.91 & 19.03 \\
\hline 240 & 63.7 & 25.68 & 38.01 \\
\hline 243 & 66.25 & 24.89 & 41.36 \\
\hline 246 & 89 & 26.33 & 62.67 \\
\hline 249 & 95.27 & 25.78 & 69.48 \\
\hline 252 & 110.25 & 26.24 & 84.02 \\
\hline 255 & 115.57 & 26.15 & 89.43 \\
\hline 258 & 109.91 & 25.64 & 84.27 \\
\hline 261 & 104.3 & 26.88 & 77.42 \\
\hline 264 & 89.5 & 26.64 & 62.86 \\
\hline 267 & 72.63 & 27.11 & 45.53 \\
\hline 270 & 73.61 & 26.55 & 47.07 \\
\hline 273 & 58.55 & 26.29 & 32.26 \\
\hline 276 & 42.62 & 26.89 & 15.73 \\
\hline 279 & 35.33 & 26.89 & 8.44 \\
\hline 282 & 39.09 & 27.05 & 12.04 \\
\hline 285 & 35.45 & 25.99 & 9.46 \\
\hline 288 & 34.38 & 26.15 & 8.24 \\
\hline 291 & 34.16 & 25.76 & 8.4 \\
\hline 294 & 33.80 & 26.84 & 6.96 \\
\hline 297 & 32.33 & 27.62 & 4.71 \\
\hline
\end{tabular}

Table F.5: Cell 5 data 


\section{Bibliography}

[1] S. Jayanthi, K. S. Nilgiriwala, and D. Del Vecchio, "Retroactivity controls the temporal dynamics of gene transcription," ACS Synthetic Biology, vol. 2, pp. 431-441, 2013.

[2] D. Mishra, P. M. Rivera, A. Lin, D. Del Vecchio, and R. Weiss, "A load driver device for engineering modularity in biological networks," Nature Biotechnology, vol. 32, pp. 1268-1275, 2014.

[3] D. Del Vecchio, A. J. Ninfa, and E. D. Sontag, "Modular cell biology: retroactivity and insulation," Nature/EMBO Molecular Systems Biology, vol. 4:161, 2008.

[4] T. S. Gardner, C. R. Cantor, and J. J. Collins, "Construction of a genetic toggle switch in Escherichia coli," Nature, vol. 403, pp. 339-342, 2000.

[5] D. E. Chang, S. Leung, M. R. Atkinson, A. Reifler, D. Forger, and A. J. Ninfa, "Building biological memory by linking positive feedback loops," Proceedings of the National Academy of Sciences of the United States of America, vol. 107, pp. 175-180, 2010.

[6] B. P. Kramer, A. U. Viretta, M. D. Baba, D. Aubel, W. Weber, and M. Fussenegger, "An engineered epigenetic transgene switch in mammalian cells," Nature Biotechnology vol. 22, pp. 867-870, 2004.

[7] M. B. Elowitz and S. Leibler, "A synthetic oscillatory network of transcriptional regulators," Nature, vol. 403, pp. 339-342, 2000. 
[8] M. Tigges, T. T. Marquez-Lago, J. Stelling, and M. Fussenegger, "A tunable synthetic mammalian oscillator," Nature, vol. 457, pp. 309-312, 2009.

[9] J. Stricker, S. Cookson, M. R. Bennett, W. H. Mather, L. S. Tsimring, and J. Hasty, "A fast, robust and tunable synthetic gene oscillator," Nature, vol. 456, pp. 516-519, 2008.

[10] J. C. Anderson, C. A. Voigt, and A. P. Arkin, "Environmental signal integration by a modular AND gate," Molecular Systems Biology, vol. 3, pp. 133, 2007.

[11] A. Tamsir, J. J. Tabor, and C. A. Voigt, "Robust multicellular computing using genetically encoded NOR gates and chemical "wires"," Nature, vol. 469, pp. 212215, 2011.

[12] D. Del Vecchio, "Modularity, context dependence, and insulation in engineered biological circuits," Trends in Biotechnology, 2014.

[13] H. Alper and G. Stephanopoulos, "Engineering for biofuels: exploiting innate microbial capacity or importing biosynthetic potential?," Nature Reviews Microbiology, vol. 7, pp. 715-723, 2009.

[14] K. J. Morey, M. S. Antunes, K. D. Albrecht, T. A. Bowen, J. F. Troupe, K. L. Havens, and J. I. Medford, "Developing a synthetic signal transduction system in plants," Methods in Enzymology, vol. 497, pp. 581-602, 2011.

[15] J. Yan et al., "Enhanced Cellular Immune Responses Elicited by an Engineered HIV-1 Subtype B Consensus-based Envelope DNA Vaccine," Molecular Therapy, vol. 15, pp. 411-421, 2007.

[16] "GENEART synthesizes genes for swine flu vaccine in record speed", Accessed at: <http://www.marketwired.com/press-release/geneart-synthesizes-genes-forswine-flu-vaccine-in-record-speed-frankfurt-g6a-1229992.htm > on April 29, 2016.

[17] T. K. Lu and J. J. Collins, "Dispersing biofilms with engineered enzymatic bacteriophage," Proceedings of the National Academy of Sciences, vol. 104, pp. 1119711202, 2007. 
[18] J. C. Anderson, E. J. Clarke, A. P. Arkin, and C. A. Voigt, "Environmentally controlled invasion of cancer cells by engineered bacteria," Journal of Molecular Biology, vol. 355, pp. 619-627, 2006.

[19] K. Nilgiriwala, J. Jimenez, P. M. Rivera, and D. Del Vecchio, "A synthetic tunable amplifying buffer circuit in E. coli," ACS Synthetic Biology, vol. 4, pp. 577-584, 2015.

[20] S. Jayanthi and D. Del Vecchio, "Retroactivity attenuation in bio-molecular systems based on timescale separation," IEEE Transactions on Automatic Control, vol. 56(4), pp. 748-761, 2011.

[21] S. Jayanthi and D. Del Vecchio, "Tuning genetic clocks employing DNA binding sites," PLOS One, vol. 7(7), e41019, 2012.

[22] S. M. Lyons, W. Xu, J. Medford, and A. Prasad, "Loads bias genetic and signaling switches in synthetic and natural systems," PLoS Computational Biology, vol. 10(3), e1003533, 2014.

[23] A. Gyorgy and D. Del Vecchio, "Modular composition of gene transcription networks," PLOS Computational Biology, vol. 10(3), e1003486, 2014.

[24] U. Alon, "An Introduction to Systems Biology: Design Principles of Biological Circuits," Chapman $\&$ Hall/CRC, 2007.

[25] BioNumbers: The database of useful biological numbers. http://bionumbers.org, 2015.

[26] I. Bendixson, "Sur les courbes définies par des équations différenetielles," Acta Mathematica, vol. 24(1), pp. 1-88, 1901.

[27] J. B. Jackson, M. Sitharam, "Descartes' Rule of Signs Revisited," American Mathematical Monthly, vol. 105, pp. 447-451, 1998.

[28] A. Mattuck, "Introduction to Analysis," Pearson Education, 2013. 
[29] C. H. Edwards, "Advanced Calculus of several variables," Dover Publications, 1994.

[30] D. del Vecchio and R. Murray, "Biomolecular Feedback Systems," Princeton University Press, 2014.

[31] K. Robison, A. M. McGuire, and G. M. Church, "A comprehensive library of DNA-binding site matrices for 55 proteins applied to the complete Escherichia coli K-12 genome," Journal of Molecular Biology, vol. 284, pp. 241-54, 1998.

[32] A. Burger, A. M. Walczak, and P. Wolynes, "Abduction and asylum in the lives of transcription factors," PNAS, vol. 107(9), pp. 4016-4021, 2010.

[33] http://oscill8.sourceforge.net/, Last accessed: March 3, 2016.

[34] R. Guantes and J. F. Poyatos, "Dynamical principles of two-component genetic oscillators," PLoS Computational Biology, 2(3): e30. doi: 10.1371/journal.pcbi.0020030, 2006.

[35] D. Del Vecchio, "Design and Analysis of an Activator-Repressor Clock in E. Coli," American Control Conference, IEEE, 1589-1594. doi: 10.1109/ACC.2007.4282275, 2007. 